\title{
Topology optimization of acoustic mechanical interaction problems: a comparative
} review

Dilgen, Cetin Batur; Dilgen, Sümer B.; Aage, Niels; Jensen, Jakob S.

Published in:

Structural and Multidisciplinary Optimization

Link to article, DOI:

10.1007/s00158-019-02236-4

Publication date:

2019

Document Version

Peer reviewed version

Link back to DTU Orbit

Citation $(A P A)$ :

Dilgen, C. B., Dilgen, S. B., Aage, N., \& Jensen, J. S. (2019). Topology optimization of acoustic mechanical interaction problems: a comparative review. Structural and Multidisciplinary Optimization, 60(2), 779-801. https://doi.org/10.1007/s00158-019-02236-4

\section{General rights}

Copyright and moral rights for the publications made accessible in the public portal are retained by the authors and/or other copyright owners and it is a condition of accessing publications that users recognise and abide by the legal requirements associated with these rights.

- Users may download and print one copy of any publication from the public portal for the purpose of private study or research.

- You may not further distribute the material or use it for any profit-making activity or commercial gain

- You may freely distribute the URL identifying the publication in the public portal 


\title{
Topology optimization of acoustic mechanical interaction problems: A comparative review
}

\author{
Cetin B. Dilgen $^{1}$. Sumer B. Dilgen ${ }^{2} \cdot$ Niels Aage $^{1} \cdot$ Jakob S. Jensen $^{1}$
}

Received: date / Accepted: date

\begin{abstract}
The pursuit for design improvements by geometry modifications can easily become prohibitive using a trial and error process. This holds especially when dealing with multi-physics problems - such as acousticstructure interaction - where it is difficult to realize design improvements intuitively due to the complexity of the coupled physics. Compared to classical shape optimization, where a near optimal shape has to be supplied as an initial guess, topology optimization allows for innovative designs through a completely free material distribution, such that the topology can change during the optimization process. The goal of this article is to provide a comprehensive critical review of the proposed strategies for topology optimization of coupled acoustic-structure interaction problems. The work includes a comparison of topology optimization formulations with density, level-set and evolutionary based methods and discusses the corresponding strengths and weaknesses through the considered application examples. The review concludes with recommendations for future research directions.
\end{abstract}

Cetin B. Dilgen and Sumer B. Dilgen contributed equally to this work.

Cetin B. Dilgen

E-mail: cedil@mek.dtu.dk

Sumer B. Dilgen

E-mail: sydil@elektro.dtu.dk

1 Centre for Acoustic-Mechanical Micro Systems (CAMM), Department of Mechanical Engineering, Technical University of Denmark, Lyngby, Denmark

${ }^{2}$ Centre for Acoustic-Mechanical Micro Systems (CAMM), Department of Electrical Engineering, Technical University of Denmark, Lyngby, Denmark
Keywords Vibro-acoustics · Topology Optimization . Density methods $\cdot$ Level set methods

\section{Introduction}

Since its introduction in the late 1980's, gradient-based topology optimization has diversified significantly both in terms of application areas, but also with emerging variants of the original design parameterization. The initial homogenization approach (Bendsøe and Kikuchi 1988) laid the foundation for the socalled density-method, often referred to as the SIMP-method (Bendsøe 1989). Here, a pixel (or in 3D: voxel) based design description is used with continuous element-wise design variables representing a relative "element density" (not to be confused with the mass density of the involved material). Elimination of intermediate-valued (grey) design variables with the penalization approach combined with appropriate regularization of the optimization problem using filtering techniques and length-scale control (Bourdin 2001, Bruns and Tortorelli|2001, Guest et al| 2004 Wang et al 2011) led to a breakthrough regarding generation of well defined (black-white) pixelized structures. The result is that only limited post-processing (if any, eg. Christiansen et al (2015)) is needed before fabrication. Combined with efficient adjoint sensitivity analysis and the use of robust and versatile mathematical programming tools, eg. The Method of Moving Asymptotes (MMA) (Svanberg 1987) this has paved the way for the tremendous popularity of this approach. Recently, a milestone has been reached with the report of giga-scale design of a full aircraft wing with more than one billion 3D elements and corresponding design variables (Aage et al 2017). Moreover, the method has also proven its worth for a number of other applications, 
ranging from material design (Larsen et al 1997), acoustics (Park and Wang 2008), optics (Jensen and Sigmund 2011), microfluidics (Alexandersen et al 2016) as well as coupled multi-physics problems such as thermo-electromechanical devices (MEMS) (Sigmund 2001), turbulent flow heat transfer systems (Dilgen et al 2018) and many other application areas.

As an inherent feature of the density-based approach, the structural boundary is constantly evolving during the optimization procedure. That is, boundaries may appear and disappear in a seamless fashion due to the continuous design variables. The corresponding structural appearance with gray scale at intermediate design stages can, however, cause uncertainty and ambiguity in identifying well defined boundaries. This poses a challenge when dealing with structures affected by eg. pressure boundary loads and calls for specialized schemes to be developed (Olhoff et al 1991; Sigmund and Clausen 2007).

The quest for a well-defined boundary has partly motivated the use of level set methods (Osher and Sethian 1988, Sethian and Wiegmann 2000) for structural topology optimization. With the aim of keeping the full design flexibility from the density-approach and combining this with the well defined boundaries known from shape optimization methods, a level set function implicitly defines the structural boundary through its zero level iso-curve. The level set function is evolved via design sensitivities and holes may merge or new holes may appear, partially thanks to the introduction of topological derivatives (Novotny and Sokołowski 2013). Several variants of the method has appeared using the original version based on a boundary-fitted mesh and design updates based on the solution of a Hamilton-Jacobi equation. Since then, many alternative versions have been introduced, of which the one based on fixed meshes, cutfem methods (Hansbo and Hansbo 2004) and the use of mathematical programming tools has been reported well-suited for complex interface problems cf. Villanueva and Maute (2017). Moreover, variants of the level set methods such as phase field methods and implicit functions have also appeared, which share theoretical and implementation details with the mentioned approaches. It should be mentioned that the numerical implementation of level set methods share many of same issues as the density method, and we refer to the review paper by Sigmund and Maute (2013) for a thorough review and comparison of the two methods for structural optimization applications.

When considering coupled multi-physics problems like electro-thermo-elastic or elasto-optical, the density and level set methods can be applied with only minor adaptation and usually without altering the ba- sic parametrization scheme. However, a challenge arises when considering coupled problems where different physical fields couple at the structural boundary. Examples include fluid-structure and acoustic-structure interaction. In this paper we focus exclusively on the acoustic-structure interaction problem, where the fluctuating acoustic pressure field acts as a boundary load on the structure and the structural vibrations act as acoustic sources. In standard solution procedures it is thus imperative to know the location of this boundary. The level set methods operate with a well-defined boundary and in both Shu et al (2014) and Desai et al (2018) the authors adapted the re-meshing based level set approach to the acoustic-structure interaction problem where the zero level set is now used to separate the structural and the acoustic domains. That is, one solves the standard structural vibration problem where the level set is positive and the acoustic Helmholtz equation is solved in regions where the level set function is negative. Instead of using re-meshing, the level set method has also been used in combination with the density parametrization, i.e. using an erstatz material model and phase field approach to drive the design evolution. In this approach, the level set field is mapped to an indicator function which in turn used to interpolate the material properties of the acoustic and elastic mediums thus preventing the challenges of tracking the boundary changes throughout the optimization. Using this technique, Noguchi et al (2015) employed the unified multiphase (UMP) technique based on Biot's theory for poroelastic waves (Lee et al 2012). In Noguchi et al (2016), the authors derived a topological derivative for a vibro-acoustic system modelled by a twophase material model. Using topological derivatives and the same modeling approach, Noguchi et al (2017) and Miyata et al (2018) carried out level set based topology optimization. The UMP technique has also been used in context of density based topology optimization of acoustic-mechanical-septa distribution by Lee et al (2015). The vibro-acoustic systems have also been studied with a combined Boundary Element (BEM) and FE formulation to take advantage of the BEM's capabilities of the modeling the unbounded acoustic domains, while relying on FEM for the structural part. Isakari et al (2017) studied the level set based topology optimization of an elastic sound scatterer using such a BEM-FEM solver.

As argued, the density method does not naturally provide knowledge of the location of the acoustic-structural interface. However, the mixed FE-formulation has been used to circumvent this problem and facilitate a monolithic density based parametrization approach. This formulation was also used in Sigmund and Clausen (2007) 
to treat the static pressure-load problem. With this formulation the pressure is introduced as an auxiliary variable in addition to the displacements which allows one to transfer pressure loads without explicit indication of the boundary location. This formulation was used in the seminal work on acoustic-structure topology optimization presented in Yoon et al (2007), and has later been applied to design a porous microstructures for increased loss factor (Kook and Jensen 2017).

In addition to these main methods we will briefly review acoustic-structure interaction problems solved by the bi-directional evolutionary topology optimization (BESO) method as presented in Picelli et al (2015), Vicente et al (2015) and Chen et al (2017). It should be noted also that a number of topology optimization problems have been studied for which the topological changes do not involve a change in the boundary between the structural and acoustic domains. The main part of these involve problems where a plate is coupled to an acoustic domain. For most problems the inplane material distribution involving two material constituents is optimized (Yamamoto et al 2008, 2009, Du and Olhoff 2010, Yang and Du 2013), but also optimal distribution of thin damping layers have been considered (Zhang and Kang 2013) in addition to optimal plate thickness distributing (Akl et al 2009), laminated composites (Niu et al 2010) and piezoelectric structures (Yoon et al 2018). Also, a few studies have appeared where the optimization problem has been defined so as to circumvent the need for modifying the structural boundaries and thus enables the use of standard parameterization schemes, see eg. Søndergaard and Pedersen (2014).

In the present paper we will conduct a review of the methods that have appeared which allows for introducing topological changes in the distribution of acoustic and structural domains, ie. the level set method, the density based method using the mixed FE-formulation, and the BESO method as a special variant of the density based method. The computational framework for the density and level set methods will be outlined and results for a series of test problems will be critically examined. All results presented will be based on the authors' implementation of the optimization algorithm. Based on this comparative review recommendations for future research will be provided.

\section{Topology optimization approaches}

The general goal of an acoustic-structure topology optimization problem is to determine the optimal layout of the structural and acoustic domains, such that a given performance measure, that we may denote $\Phi(\mathbf{u}(\mathbf{x}, t), p(\mathbf{x}, t))$, is minimized. This formulation indicates that the performance measure may depend both on both the structural vibration level (the vibration amplitude $\mathbf{u}$ ) as well as the acoustic pressure amplitude $p$.

Fig. 1(a) shows an illustration of the initial configuration for a typical acoustic-structure optimization problem. Here, a mechanical structure has two internal acoustic cavities and is surrounded by an external acoustic medium. The structure is excited by a time-harmonic mechanical load $f(t)$ and also a timeharmonic acoustic load $q(t)$. The loads generate vibrations of the structure and also acoustic pressure fluctuation in the acoustic medium. The aim of the optimization problem is now to minimize the prescribed objective function

$\min \Phi(\mathbf{u}(\mathbf{x}, t), p(\mathbf{x}, t))$

which in the hypothetical scenario illustrated in Fig. 1 results in the optimized distribution of structural and acoustic domains shown in Fig. 1(b). During the optimization process the structural domain has been reshaped and one of the internal acoustic cavities has been removed. Acoustic-structure optimization problems will most often involve additional constraints on the allowable performance or its configuration. A typical constraint in topology optimization is to enforce a maximum allowable volume of the structure, which we can formulate as:

$\frac{\int_{\Omega_{s}} d \Omega}{\int_{\Omega} d \Omega} \leq V^{*}$

where $\Omega_{s}$ is the domain occupied by the structure, $\Omega$ is the total domain under consideration, and $V^{*}$ is the allowable volume fraction.

In order to solve this optimization problem the following key points should be addressed:

- Design parametrization. The methods reviewed apply parametrization based on the density or the level set approach.

- Analysis method. All strategies reviewed rely on finite element analysis of the underlying structural and acoustic problems. The formulations used depend on the choice of parametrization applying either a standard segregated analysis combined with a body-fitted and adaptive FE-mesh (level set approach) or a mixed formulation combined with a fixed and regular FE-mesh (density approach).

- Sensitivity analysis. The methods reviewed are gradientbased (the BESO method only to a certain extend) and apply sensitivity analysis based on the FE analysis. 

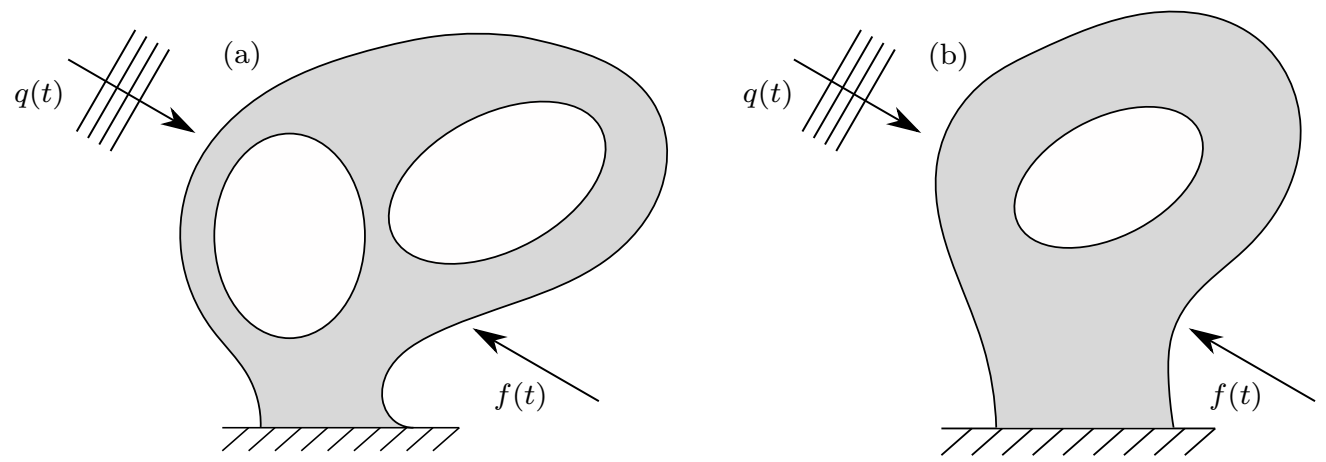

Fig. 1: Schematic illustration of a typical optimization problem: (a) Initial configuration (b) Optimized configuration.

- Design updates. The choice of design update method is also linked to the choice of parametrization scheme: The works using the level set approach use an update scheme based on a solution to the HamiltonJacobi transport equation. The density approach uses mathematical programming e.g. MMA, while the BESO uses a heuristic update scheme.

In the following the strategies outlined in the four key points will be described in details including implementation considerations.

\subsection{Design parametrization}

Existing approaches for topology optimization of acousticstructure interaction problems can, as previously stated, be split into two main groups depending on the choice of design parametrization. Note however, that although the design representations used for the density and level set approaches are conceptually very different, it can be argued that when it comes to a numerical implementation, the similarities actually outweigh the differences as reported for the case of structural optimization in Sigmund and Maute (2013).

\subsubsection{The density approach}

The basic idea of density based structural optimization is to describe the topology by a spatially varying design field, here $\gamma(\mathbf{x})$. In the discretized setting this is achieved by assigning a design variable to each of the computational domain pixels (2D) or voxels (3D). For the case of acoustic-structure interaction this means that a pixel with $\gamma=1$ is interpreted as being structure, while $\gamma=0$ corresponds to an acoustic medium. The only difference to classical structural topology optimization is that $\gamma=0$ corresponds to void in that case. Also, it is worth noting that in the density method the same mesh is often used for both analysis and design and the mesh is usually kept fixed throughout the optimization process. Furthermore, the mesh is often regular which means that elaborate mesh generation or re-meshing is avoided. In order to apply gradient based optimization methods the design variable field is represented as continuous, ie.

$0 \leq \gamma_{i} \leq 1$

where $i$ indicates the pixel (element) number.

A schematic of the entire density based optimization process is illustrated in Fig. 2 Fig. 2 (a) shows a possible initial structure modelled by setting the appropriate design variables to either 1 (black) or 0 (white). However, one of the greatest strengths of the density approach is that initial designs are often not needed. In fact, more often a uniform distribution of the design variables is applied as illustrated in Fig. 2(b). The choice of the initial design distribution is in general arbitrary, but could be chosen to match the volume constraint $\gamma_{i}=V^{*}$, if such a constraint is present. Naturally, a random distribution of the densities can be applied as well. As the optimization process progresses, the topology of the design evolves and often grey regions appear as shown in Fig. 2(c). Through different filtering and projection methods, the intermediate design variables are slowly suppressed until the design converges to a completely black (1) or white (0) configuration. The optimized design is illustrated in Fig. 2(d) which also shows how the pixel based representation leads to the so-called staircasing phenomena. That is, although the design is completely black and white, the interface between solid and acoustic region is jagged. This might pose problems in multiphysics settings, which is why it is sometimes beneficial to allow the interface to be smeared over a couple of pixels. 
(a)
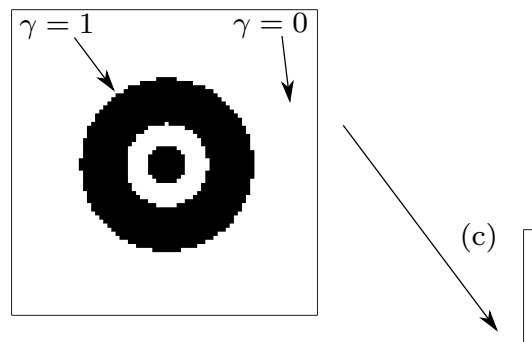

$0 \leq \gamma \leq 1$

(b)

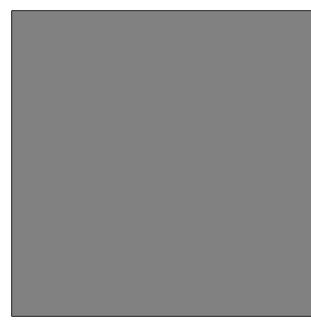

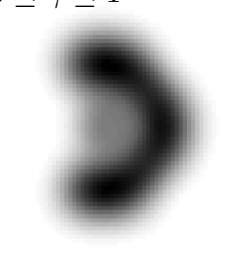

$(\mathrm{d})$

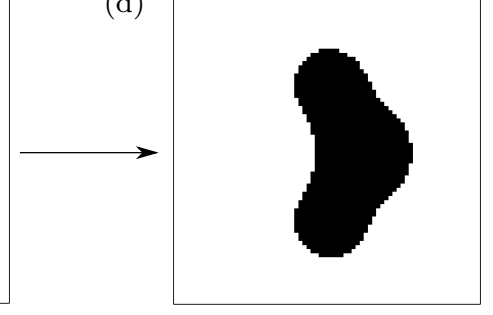

Fig. 2: Illustration of parametrization using the density approach. (a) Initial design using a design configuration, (b) initial design with homogeneous distribution of pixel densities, (c) intermediate design with grey pixels, (d) optimized design.

\subsubsection{The level set approach}

In level set based topology optimization the design representation is most often separated from the underlying analysis mesh. This is achieved by representing the topology by a scalar function $\phi$, and by defining the different material phases and interface based on the following rule

$$
\begin{aligned}
& \phi(x)>0, \quad x \in \Omega_{s} \text { (structural domain) } \\
& \phi(x)=0, \quad x \in \partial \Omega \text { (interface) } \\
& \phi(x)<0, \quad x \in \Omega_{a} \text { (acoustic domain) }
\end{aligned}
$$

Fig. 3(a) illustrates an example of a level set function and how it can be used to define structural and acoustic domains as well as their common interface. This definition is a straightforward extension of the approach usually used for structural topology optimization where $\phi<0$ then corresponds to void. The level set function is usually discretized on a regular grid, similar to that for the design variables in the density based method. However, the level set values are most often assigned to nodal points instead of element centers.

Fig. 4(a) shows a representative level set function discretized on a regular grid, whereas Fig 4 (b) shows the corresponding design configuration described by the the zero-level contour. During the optimization process, the level set function evolves into a new configuration. This is illustrated in Figs. 4(c) and 4(d) which shows the grid based level set function and its geometric interpretation, respectively.
The mapping of the level set function to the mesh used for analysis can be done in several ways. The method most often used in structural optimization is to apply an ersatz material model and in this case the level set method and the density based method are very similar with only minor differences. To the author's knowledge this approach has not yet been applied to acoustic-structure interaction problems. Instead, the approach used in the existing works is to perform a complete remeshing based on the zero level contour, which ensures a crisp and well-defined interface. However, we should note that recent work on level set based topology optimization applies novel immersed boundary methods such as cut finite elements or finite cell representations. These methods allow the analysis and level meshes to coincide, while still maintaining the crisp interface by using elaborate integration schemes (see e.g. Hansbo and Hansbo (2004) and Düster et al (2008) for more).

Implicit representation of the interface using the values of the level set function $\phi(x)$ also allows for easy calculation of the geometric properties such as the unit normal vector and the mean curvature, not only on the design interface $\partial \Omega$, but everywhere in the domain $D$. The unit normal vector $\mathbf{n}_{a}$ pointing out from the acoustic domain is calculated as

$\mathbf{n}_{a}=\frac{\nabla \phi}{|\nabla \phi|}$ 


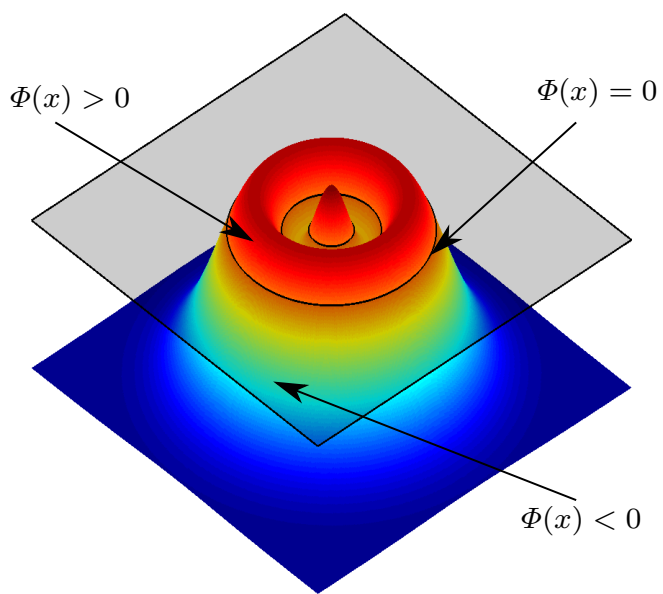

(a)

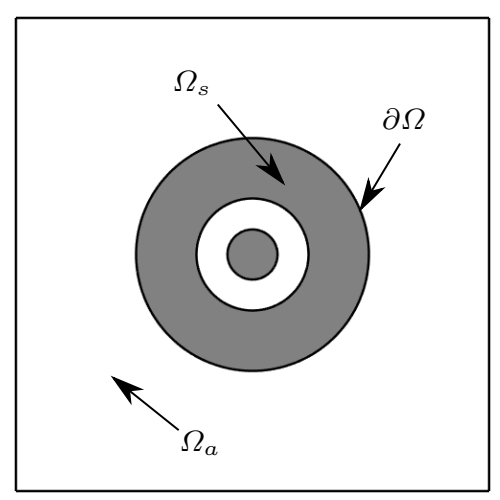

(b)

Fig. 3: Level set model and the design domain: (a) level set function and its zero level set; (b) $\Omega_{s}$ and its embedding domain $\Omega$.

(a)

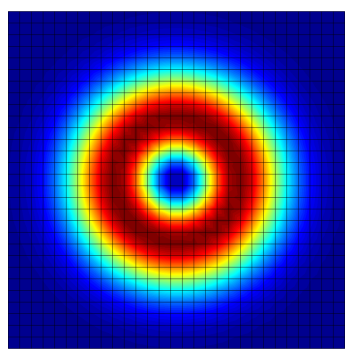

Optimization Process

(c)
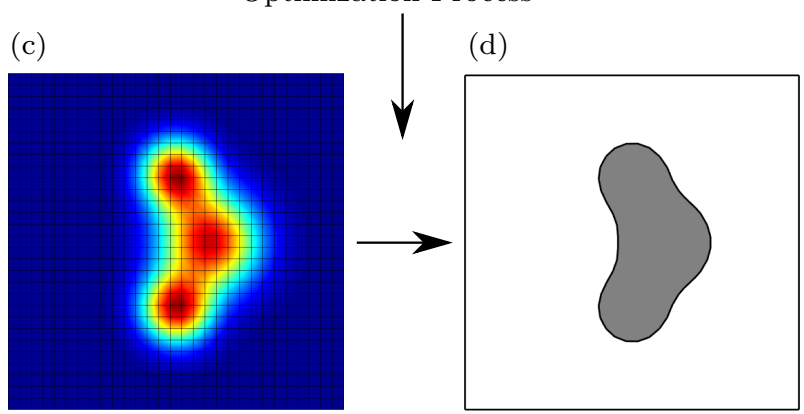

Fig. 4: Illustration of the discretized level set function, (a) Grid values of the initial level set function, (b) initial design described by the the zero-level iso-curve, (c) Grid values of the optimized level set function, (d) final design described by the the zero-level iso-curve.

Likewise the calculation of the mean curvature is also tied to the level set function

$$
\begin{aligned}
\kappa & =\nabla \cdot \mathbf{n}_{a} \\
& =\nabla \cdot\left[\frac{\nabla \phi}{|\nabla \phi|}\right]
\end{aligned}
$$

2.2 Finite element analysis of acoustic-structural interaction (ASI)

Discretizing and meshing the analysis domain based on the zero-level contour of the scalar level set function, makes it possible to employ standard segregated analysis. That is, the structural equation is solved only in the solid region and the acoustic wave equation is solved only in the acoustic region. Thus, this approach is analogous to standard simulation of acoustic-mechanical interaction problems as used in most commercial and open source numerical tool boxes. In the following section we will describe how to perform the segregated analysis using standard finite elements.

\subsubsection{Segregated analysis}

The governing equations for the time-harmonic motion of a linear elastic body $\Omega_{s}$ can be written as

$$
\begin{aligned}
& \nabla^{T} \boldsymbol{\sigma}+\omega^{2} \rho_{s} \overline{\mathbf{u}}=0 \quad \text { in } \quad \Omega_{s} \\
& \boldsymbol{\sigma}=\mathcal{C} \boldsymbol{\epsilon} \\
& \boldsymbol{\epsilon}=\left\{\epsilon_{11} \epsilon_{22} 2 \epsilon_{12}\right\}^{T} \quad(2 \mathrm{D}) \\
& \epsilon_{11}=\frac{\partial \bar{u}_{1}}{\partial x}, \quad \epsilon_{22}=\frac{\partial \bar{u}_{2}}{\partial y}, \quad \epsilon_{12}=\frac{1}{2}\left(\frac{\partial \bar{u}_{1}}{\partial y}+\frac{\partial \bar{u}_{2}}{\partial x}\right)
\end{aligned}
$$

where body forces have been neglected, $\rho_{s}$ is the density of the solid and $\omega$ is the radial frequency. Moreover, $\boldsymbol{\sigma}$ is the Cauchy stress vector, $\mathcal{C}$ is the constitutive matrix and the $\boldsymbol{\epsilon}$ is the strain vector. We consider the body to be subjected to standard boundary conditions as well as being adjacent to an acoustic medium in a part of 


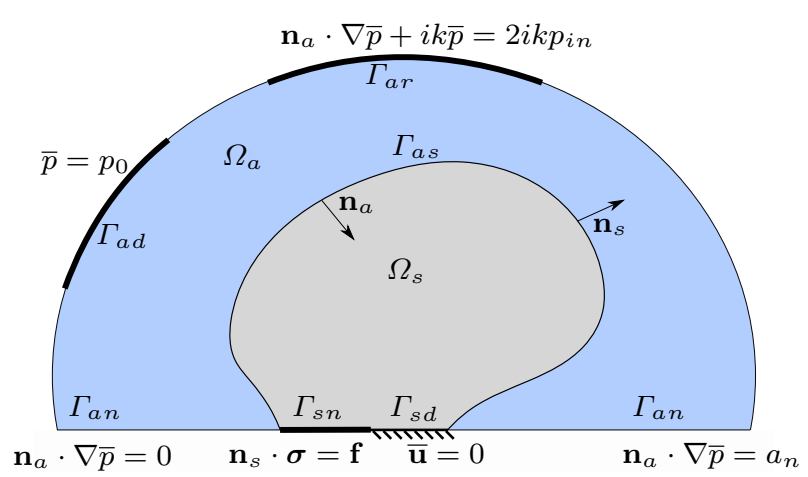

Fig. 5: The coupled acoustic-structure system: the acoustic fluid domain $\Omega_{a}$ and the structural domain $\Omega_{s}$ coupled by integrals over the acoustic-structure interface $\Gamma_{a s}$.

the boundary. The boundary conditions read

$\overline{\mathbf{u}}=\mathbf{u}_{0} \quad$ in $\quad \Gamma_{s d}$

$\mathbf{n}_{s}^{T} \boldsymbol{\sigma}=\mathbf{f} \quad$ in $\quad \Gamma_{s n}$

$\mathbf{n}_{s}^{T} \boldsymbol{\sigma}=p \mathbf{n}_{a} \quad$ in $\quad \Gamma_{a s}$

where $\mathbf{n}_{a}$ is the normal vector of the acoustic boundary pointing outward from the acoustic domain defined in Eq. (5) and $\mathbf{n}_{s}$ is the normal vector of the structural boundary pointing to the acoustic domain. The vector $\mathbf{f}$ is the traction force defined on the part of the structural boundary $\Gamma_{s n}$. The definitions for the coupled system is illustrated in Fig. 5 including all boundary conditions used throughout the presented work.

Similarly, the strong form of the Helmholtz equation describing the pressure fluctuations in the acoustic domain is written as

$\nabla^{2} \bar{p}+\left(\frac{\omega}{c_{a}}\right)^{2} \bar{p}=0 \quad$ in $\quad \Omega_{a}$

along with the following common boundary conditions:

$\bar{p}=p_{0} \quad$ in $\quad \Gamma_{a d}$

$\mathbf{n}_{a}^{T} \nabla \bar{p}=a_{n} \quad$ in $\quad \Gamma_{a n}$

$\mathbf{n}_{a}^{T} \nabla \bar{p}=-\omega^{2} \rho_{a} \mathbf{n}_{s}^{T} \overline{\mathbf{u}} \quad$ in $\quad \Gamma_{a s}$

$\mathbf{n}_{a}^{T} \nabla \bar{p}+\mathrm{i} k \bar{p}=2 \mathrm{i} k p_{i n} \quad$ in $\quad \Gamma_{a r}$

where $c_{a}$ is the speed of sound in the acoustic medium, $\rho_{a}$ is the density, $k=\frac{\omega}{c_{a}}$ is the wave number, $\mathrm{i}$ is the imaginary unit and $a_{n}$ is the prescribed Neumann boundary condition which is zero for the hard wall boundaries.

Applying the standard Galerkin procedure to carry out the finite element discretization of the governing equations, the following weak form of the elasticity equation is obtained

$$
\begin{array}{r}
\int_{\Omega_{s}} \delta \boldsymbol{\epsilon}^{T} \mathcal{C} \boldsymbol{\epsilon} \mathrm{d} \Omega-\omega^{2} \rho_{s} \int_{\Omega_{s}} \delta \overline{\mathbf{u}}^{T} \overline{\mathbf{u}} \mathrm{d} \Omega \\
-\int_{\Gamma_{a s}} \delta \overline{\mathbf{u}}^{T} \bar{p} \mathbf{n}_{a} \mathrm{~d} \Gamma=\int_{\Gamma_{s n}} \delta \overline{\mathbf{u}}^{T} \mathbf{f} \mathrm{d} \Gamma
\end{array}
$$

Similarly, the weak form of the acoustic domain reads

$$
\begin{aligned}
& \int_{\Omega_{a}}(\nabla \delta \bar{p})^{T} \nabla \bar{p} \mathrm{~d} \Omega-\frac{\omega^{2}}{c_{a}^{2}} \int_{\Omega_{a}} \delta \bar{p} \bar{p} \mathrm{~d} \Omega \\
& -\omega^{2} \rho_{a} \int_{\Gamma_{a s}} \delta \bar{p} \mathbf{n}_{a}^{T} \overline{\mathbf{u}} \mathrm{d} \Gamma=\int_{\Gamma_{a n}} \delta \bar{p} a_{n} \mathrm{~d} \Gamma
\end{aligned}
$$

where the radiation boundary condition in Eq. 18 is excluded from the derivation for brevity. Here, $\delta \boldsymbol{\epsilon}$ is the virtual strain, $\delta \overline{\mathbf{u}}$ and $\delta \bar{p}$ are the test functions for displacements and the pressure field, respectively. In order to carry out the discretization, the continuous variables $\overline{\mathbf{u}}$ and $\bar{p}$ are approximated using linear isoparametric shape functions

$$
\begin{aligned}
& \overline{\mathbf{u}}=\mathbf{N}_{u} \mathbf{u} \\
& \bar{p}=\mathbf{N}_{p} \mathbf{p} \\
& \boldsymbol{\epsilon}=\boldsymbol{\partial} \mathbf{N}_{u} \mathbf{u}=\mathbf{B}_{u} \mathbf{u}
\end{aligned}
$$

where $\boldsymbol{\partial}$ is the differential operator and $\mathbf{B}_{u}$ is the linear strain-displacement matrix. The discretized matrix system of the weak form is then

$$
\left[\begin{array}{cc}
\left(\mathbf{K}_{s}-\omega^{2} \mathbf{M}_{s}\right) & -\mathbf{C} \\
-\rho_{a} \omega^{2} \mathbf{C}^{T} & \left(\mathbf{K}_{a}-\omega^{2} \mathbf{M}_{a}\right)
\end{array}\right]\left[\begin{array}{l}
\mathbf{u} \\
\mathbf{p}
\end{array}\right]=\left[\begin{array}{l}
\mathbf{f} \\
\mathbf{g}
\end{array}\right]
$$

with the matrices and vectors being

$$
\begin{aligned}
& \mathbf{K}_{s}=\int_{\Omega_{s}} \mathbf{B}_{u}^{T} \mathcal{C} \mathbf{B}_{u} \mathrm{~d} \Omega_{s} \\
& \mathbf{M}_{s}=\int_{\Omega_{s}} \rho_{s} \mathbf{N}_{u}^{T} \mathbf{N}_{u} \mathrm{~d} \Omega_{s} \\
& \mathbf{f}=\int_{\Gamma_{s n}} \mathbf{N}_{u}^{T} \mathbf{f} \mathrm{d} \Gamma_{s n}
\end{aligned}
$$

for the structural domain and

$$
\begin{aligned}
& \mathbf{K}_{a}=\int_{\Omega_{a}} \mathbf{B}_{p}^{T} \mathbf{B}_{p} \mathrm{~d} \Omega_{a} \\
& \mathbf{M}_{a}=\int_{\Omega_{a}} \frac{1}{c_{a}^{2}} \mathbf{N}_{p}^{T} \mathbf{N}_{p} \mathrm{~d} \Omega_{a} \\
& \mathbf{g}=\int_{\Gamma_{a n}} \mathbf{N}_{p}^{T} a_{n} \mathrm{~d} \Gamma_{a n}
\end{aligned}
$$

for the acoustic domain where the matrix $\mathbf{B}_{p}=\boldsymbol{\partial} \mathbf{N}_{p}$. The coupling matrix is found as:

$\mathbf{C}=\int_{\Gamma_{a s}} \mathbf{N}_{u}^{T} \mathbf{n}_{a} \mathbf{N}_{p} \mathrm{~d} \Gamma_{a s}$ 
(a)

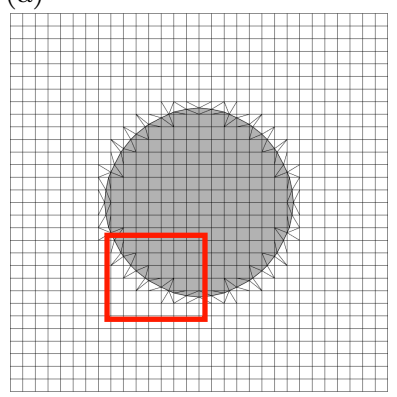

(b)

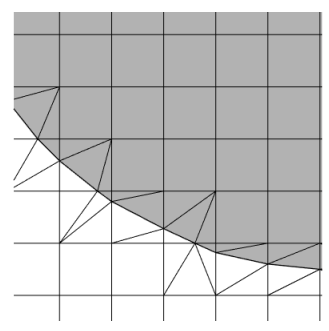

Fig. 6: Illustration of a fitted finite element mesh to realize the boundary curve. (a) Whole computation mesh, (b) Close up view of the mesh showing the triangulation.

As previously mentioned the mesh is usually adapted to the acoustic-structural boundary in each step of the optimization algorithm. Fig. 6 shows an example of a such finite element grid where the the boundary curve is obtained utilizing the marching square algorithm and the curve is fitted to the mesh using simple triangulation.

\subsubsection{Mixed formulation}

In the case of a density based parametrization, it is no longer possible to clearly define the acoustic and structural parts of the domain due to the possible appearance of "grey" pixels in the design. Therefore a single monolithic formulation governing both physics and their coupling is needed. The mixed formulation has been proposed to do exactly this, that is, to model the acoustic-mechanical interaction problems without having an explicit boundary representation. The mixed finite element formulation, also called the $\mathbf{u} / p$ (displacement/pressure) formulation, can be found in many references, see eg. Zienkiewicz and Taylor (2000). In Wang and Bathe (1997) the formulation was proposed to model acoustic-mechanical interaction problems. In Sigmund and Clausen (2007), the static mixed FE-formulation was used to solve pressure load problems in densitybased topology optimization and in Yoon et al (2007) the formulation was used for the first time for topology optimization of acoustic-structure interaction problems.

In the following we present and motivate the use of the mixed formulation for density based topology optimization. The mixed formulation is derived by first defining a pressure variable using the bulk modulus as follows

$\bar{p}=-K \mathbf{m}^{T} \boldsymbol{\epsilon}$ which is valid for small strains. Here, $K$ is the bulk modulus and $\mathbf{m}=\{1,1,0\}^{T}$ is the vector formulation for the Kronecker's delta considering two dimensional analysis. The stress-strain relationship can then be stated as

$\boldsymbol{\sigma}=2 G \mathbf{e}-\mathbf{m} \bar{p}$

where $G$ is the shear modulus and the deviatoric strain vector e reads

$\mathbf{e}=\left(\mathbf{I}_{0}-\frac{1}{2} \mathbf{m m}^{T}\right) \boldsymbol{\epsilon}$

with $\mathbf{I}_{0}$ defined as a diagonal matrix $\mathbf{I}_{0}=\operatorname{diag}(1,1,0.5)$. Moreover, the bulk and shear moduli are defined from the Young's modulus $E$ and the Poisson's ratio $\nu$ as

$K=\frac{E}{2(1-\nu)}, \quad G=\frac{E}{2(1+\nu)}$

in the case of $2 \mathrm{D}$ plane stress conditions. The coupled equations derived from the mixed formulation can now be written as

$\nabla^{T}(2 G \mathbf{e}-\mathbf{m} \bar{p})+\omega^{2} \rho \overline{\mathbf{u}}=0$

$\frac{\bar{p}}{K}+\mathbf{m}^{T} \boldsymbol{\epsilon}=0$

which can be viewed as an alternative formulation of the original structural equations (with the advantage that it can be applied to perfectly incompressible materials as well).

However, it can also be shown that the Helmholtz equation governing the acoustic pressure can be recovered from this equation by substituting $\overline{\mathbf{u}}$ in Eq. into Eq. (34) and setting the shear modulus to zero $(G=0)$. Thus, using the mixed formulation, structural $\Omega_{s}$ and acoustic $\Omega_{a}$ parts of the domain $\Omega$ can be realized by defining the bulk modulus $K$, shear modulus $G$ and density $\rho$ as:

$K=K_{s}, \quad G=G_{s}, \quad \rho=\rho_{s} \quad$ on $\Omega_{s}$

$K=K_{a}, \quad G=0, \quad \rho=\rho_{a} \quad$ on $\Omega_{a}$

In regions with intermediate values of $\gamma$, thus not belonging to neither $\Omega_{s}$ nor $\Omega_{a}$, it is proposed to interpolate the values of $K, G$ and $\rho$ between the structural and acoustic values in Eq. (35). Such an interpolation is found in Yoon et al (2007):

$K(\gamma)=K_{s} \frac{\gamma}{1+(1-\gamma) n}+K_{a}\left(1-\frac{\gamma}{1+(1-\gamma) n}\right)$

$G(\gamma)=G_{s} \frac{\gamma}{1+(1-\gamma) n}$

$\rho(\gamma)=\rho_{s} \gamma+\rho_{a}(1-\gamma)$

in which the interpolation of $K$ and $G$ is based on a two material RAMP interpolation scheme (Stolpe and 


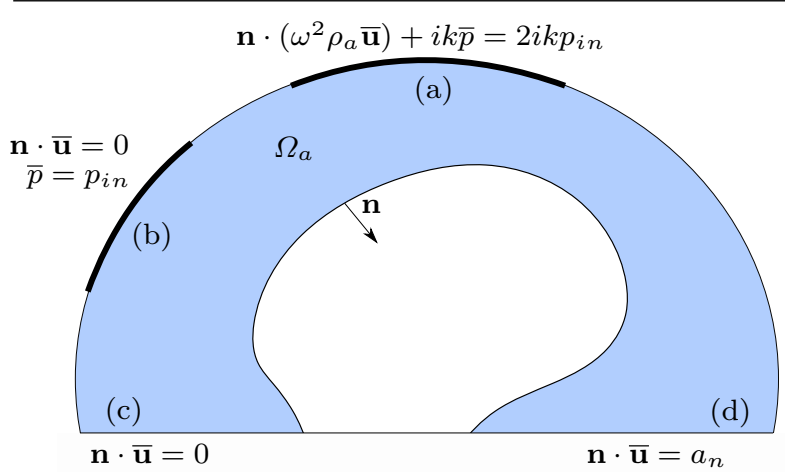

Fig. 7: Boundary conditions for the mixed finite element method in acoustic domain $\Omega_{a}$. (a) Radiation, (b) dirichlet, (c) hard wall, (d) acceleration.

Svanberg 2001) used to avoid the artificial vibration modes in low density areas reported for the more standard SIMP interpolation function (Pedersen 2000). Here, the parameter $n$ is a positive number that controls the curvature of the interpolation function. As seen from the Eq. (38), the mass density $\rho$ is interpolated linearly between the acoustic and the structural domains.

Acoustic boundary conditions for the mixed formulation are derived using that $\nabla \bar{p}=\omega^{2} \rho_{s} \overline{\mathbf{u}}$. Fig. 7 illustrates the most commonly used boundary conditions for the mixed formulation in the acoustic domain. Boundary conditions for the structural domain remain the same and do not require any special attention.

The weak form of the mixed formulation given in Eqs. (33) and (34) are obtained following the standard Galerkin approach

$\int_{\Omega} \delta \boldsymbol{\epsilon}^{T} 2 G \mathbf{D} \boldsymbol{\epsilon} \mathrm{d} \Omega-\int_{\Omega} \delta \boldsymbol{\epsilon}^{T} \mathbf{m} \bar{p} \mathrm{~d} \Omega-\omega^{2} \rho \int_{\Omega} \delta \overline{\mathbf{u}}^{T} \overline{\mathbf{u}} \mathrm{d} \Omega=0$

$\int_{\Omega} \delta \bar{p} \frac{\bar{p}}{K} \mathrm{~d} \Omega+\int_{\Omega} \delta \bar{p} \mathbf{m} \epsilon \mathrm{d} \Omega=0$

where $\delta \boldsymbol{\epsilon}$ is the virtual strain, $\delta \overline{\mathbf{u}}$ and $\delta \bar{p}$ are the test functions for the displacement and the pressure field, respectively. Moreover, the matrix $\mathbf{D}$ is defined as

$\mathbf{D}=\mathbf{I}_{0}-\frac{1}{2} \mathbf{m m}^{T}$

Again the continuous variables $\overline{\mathbf{u}}$ and $\bar{p}$ are approximated by the following shape functions

$\overline{\mathbf{u}}=\mathbf{N}_{u} \mathbf{u}$

$\bar{p}=\mathbf{N}_{p} \mathbf{p}$

$\boldsymbol{\epsilon}=\partial \mathbf{N}_{u} \mathbf{u}=\mathbf{B}_{u} \mathbf{u}$

where $\boldsymbol{\partial}$ is the differential operator and $\mathbf{B}_{u}$ is the linear strain-displacement matrix. Inserting the approximation in Eq. (42), into the weak forms in Eqs. (39) and
(40), a matrix formulation of the weak form is obtained as

$\left[\begin{array}{cc}\left(\mathbf{K}-\omega^{2} \mathbf{M}\right) & -\mathbf{C} \\ -\mathbf{C}^{T} & -\mathbf{A}\end{array}\right]\left[\begin{array}{l}\mathbf{u} \\ \mathbf{p}\end{array}\right]=\left[\begin{array}{l}\mathbf{f} \\ \mathbf{g}\end{array}\right]$

where

$\mathbf{K}=\int_{\Omega} 2 G \mathbf{B}_{u}^{T} \mathbf{D} \mathbf{B}_{u} \mathrm{~d} \Omega$

$\mathbf{M}=\int_{\Omega} \rho \mathbf{N}_{u}^{T} \mathbf{N}_{u} \mathrm{~d} \Omega$

$\mathbf{C}=\int_{\Omega} \mathbf{B}_{u}^{T} \mathbf{m} \mathbf{N}_{p} \mathrm{~d} \Omega$

$\mathbf{A}=\int_{\Omega} \frac{1}{K} \mathbf{N}_{p}^{T} \mathbf{N}_{p} \mathrm{~d} \Omega$

In order to realize a stable finite element solution to the mixed $\mathbf{u} / p$ formulation, displacement variables should use higher order interpolations than the auxiliary pressure variable (Zienkiewicz and Taylor 2000, Wang and Bathe 1997). To this end, displacement variables are discretized with second order Lagrangian shape functions whereas the pressure field is represented using first order Lagrangian shape function. For triangular and quadrilateral elements, this corresponds to using $T 6 / 3$ and $Q 8 / 4$ elements, respectively.

\subsection{Sensitivity analysis}

A gradient-based optimization approach requires computation of the sensitivities of the objective function and constraints with respect to the design variables. In the following we will outline the basic procedure followed when using the density and the level set ap0 proaches.

\subsubsection{Density-based parametrization}

In order to carry out the adjoint analysis, firstly the Lagrangian is formed

$\mathcal{L}=\Phi(\boldsymbol{\gamma}, \mathbf{v}(\gamma))+\boldsymbol{\lambda}^{T} \mathbf{r}(\boldsymbol{\gamma}, \mathbf{v}(\boldsymbol{\gamma}))$

where $\mathbf{r}=\mathbf{S v}-\mathbf{h}=0$ defines the residual vector, $\mathbf{v}=\{\mathbf{u}, \mathbf{p}\}^{T}$ is the vector of state variables, $\mathbf{h}=\{\mathbf{f}, \mathbf{g}\}^{T}$ is the source vector, $\mathbf{S}$ is the system matrix found from Eq. (43), and $\boldsymbol{\lambda}$ is a vector of Lagrange multipliers. For zero residual the Lagrangian coincides with the objective function. The derivative with respect to the design variable can be written following the chain rule as

$$
\begin{aligned}
\frac{\mathrm{d} \mathcal{L}}{\mathrm{d} \boldsymbol{\gamma}} & =\frac{\partial \Phi}{\partial \boldsymbol{\gamma}}+\frac{\partial \Phi}{\partial \mathbf{v}} \frac{\partial \mathbf{v}}{\partial \boldsymbol{\gamma}}+\boldsymbol{\lambda}^{T}\left(\frac{\partial \mathbf{r}}{\partial \boldsymbol{\gamma}}+\frac{\partial \mathbf{r}}{\partial \mathbf{v}} \frac{\partial \mathbf{v}}{\partial \boldsymbol{\gamma}}\right) \\
& =\frac{\partial \Phi}{\partial \boldsymbol{\gamma}}+\boldsymbol{\lambda}^{T} \frac{\partial \mathbf{r}}{\partial \boldsymbol{\gamma}}+\underbrace{\left(\frac{\partial \Phi}{\partial \mathbf{v}}+\boldsymbol{\lambda}^{T} \frac{\partial \mathbf{r}}{\partial \mathbf{v}}\right)}_{=0} \frac{\partial \mathbf{v}}{\partial \boldsymbol{\gamma}}
\end{aligned}
$$


Since the Lagrange multiplier can be freely chosen, it is selected such that the underlined part of the equation becomes zero to avoid the computationally expensive evaluation of the term $\frac{\partial \mathbf{v}}{\partial \boldsymbol{\gamma}}$. The adjoint equation then becomes

$\mathbf{S}^{T} \boldsymbol{\lambda}=-\left(\frac{\partial \Phi}{\partial \mathbf{v}}\right)^{T}$

Here it is noted that, the state variables can be complex variables if eg. the radiation condition given in Fig. 7 is utilized. In this case, the term $\frac{\partial \Phi}{\partial \mathbf{v}}$ on the right hand side of the adjoint equation in Eq. (50) is realized as (Dühring et al 2008)

$\frac{\partial \Phi}{\partial \mathbf{v}}=\frac{\partial \Phi}{\partial \mathbf{v}_{r}}-\mathrm{i} \frac{\partial \Phi}{\partial \mathbf{v}_{i}}$

where the subscripts $r$ and $i$ denote the real and the imaginary parts of a complex number. Having calculated the Lagrange multipliers that satisfy Eq. (50), the sensitivity of the objective function is then calculated as

$\frac{\mathrm{d} \Phi}{\mathrm{d} \boldsymbol{\gamma}}=\frac{\partial \Phi}{\partial \boldsymbol{\gamma}}+\Re\left(\boldsymbol{\lambda}^{T} \frac{\partial \mathbf{S}}{\partial \boldsymbol{\gamma}} \mathbf{v}\right)$

where $\Re(\cdot)$ denotes the real part of a complex quantity. The $\Re(\cdot)$ operator is used to compress the sensitivity equation into a simplified form and details of this derivation can be extracted from the results in Jensen (2007).

It should be emphasized that the adjoint approach presented here is especially efficient for a very high number of design variables and a low number of constraint functions. The main cost is associated with solving the adjoint equation Eq. 50 - a step that is particularly cheap if the original FE equation is solved using a direct solver since the stiffness matrix is then already factorized.

\subsubsection{Level set parametrization}

Level set sensitivity analysis differs significantly from the density-based analysis presented in section 2.3.1 where the differentiation was carried out with respect to an element design variable $\gamma$. Instead the derivatives are here handled by treating the domain $\Omega$ as a continuous medium and examining what happens when the boundary $\partial \Omega$ is perturbed, ie. the sensitivities take the form of shape derivatives. However, there are also similarities, since the adjoint method is employed for efficient computation. An in depth introduction to shape derivatives can be found in Choi and Kim (2005). Here it is noted that we have left out the overbar notation for the continuous variables to keep the notation clean for the reminder of this work.

We will assume, without loss of generality, that the objective function to be minimized can be written on the following form:

$\mathcal{J}=\int_{\Omega_{o b j}} \Phi(\mathbf{u}, p) \mathrm{d} \Omega$

The shape derivative of the objective function $\mathcal{J}$ is now computed as

$$
\begin{aligned}
\dot{\mathcal{J}}=\int_{\Omega_{o b j}} \frac{\partial \Phi}{\partial p} p^{\prime} \mathrm{d} \Omega & +\int_{\Omega_{o b j}} \frac{\partial \Phi}{\partial \mathbf{u}} \mathbf{u}^{\prime} \mathrm{d} \Omega \\
& +\int_{\partial \Omega_{o b j}} \Phi V_{n} \mathrm{~d} \Gamma
\end{aligned}
$$

where the prime superscript specifies a derivative with respect to a pseudo-time, $\partial \Omega$ is the boundary variation and $V_{n}$ represents a normal (or design) velocity of the boundary. For simplicity we will assume that $\Omega_{o b j}$ is a fixed domain and separate from the design domain. Hence, the third term in Eq. (54) vanishes because $V_{n}=$ 0 for $\partial \Omega_{o b j}$ since there is no overlap between the design and objective domains.

In a similar way as for the density based sensitivity analysis we will construct an adjoint problem in order to avoid explicit computation of the terms $p^{\prime}$ and $\mathbf{u}^{\prime}$. For this purpose a weak form of the governing equations is constructed, where the test functions are replaced by Lagrange's multipliers $\lambda_{p}$ and $\boldsymbol{\lambda}_{u}$, corresponding to the pressure and displacement fields, respectively. Furthermore, the two weak forms are added into one single equation:

$$
\begin{aligned}
& \int_{\Omega_{s}} \boldsymbol{\lambda}_{\epsilon}^{T} \mathcal{C} \epsilon \mathrm{d} \Omega-\omega^{2} \rho_{s} \int_{\Omega_{s}} \boldsymbol{\lambda}_{u}^{T} \mathbf{u} \mathrm{d} \Omega \\
& +\int_{\Omega_{a}}\left(\nabla \lambda_{p}\right)^{T} \nabla p \mathrm{~d} \Omega-\frac{\omega^{2}}{c_{a}^{2}} \int_{\Omega_{a}} \lambda_{p} p \mathrm{~d} \Omega \\
& -\int_{\Gamma_{a s}} \lambda_{u}^{T} \mathbf{n}_{a} p \mathrm{~d} \Gamma-\omega^{2} \rho_{a} \int_{\Gamma_{a s}} \lambda_{p} \mathbf{n}_{a}^{T} \mathbf{u} \mathrm{d} \Gamma=0
\end{aligned}
$$

where $\boldsymbol{\lambda}_{\epsilon}$ has also been introduced as an adjoint strain field which is calculated as $\boldsymbol{\lambda}_{\epsilon}=\mathbf{B}_{u} \boldsymbol{\lambda}_{u}$. In Eq. (55) the radiation boundary $\Gamma_{a r}$ is not included and the Neumann boundaries for both acoustic and structural domains $\left(\Gamma_{a n}\right.$ and $\left.\Gamma_{s n}\right)$ are considered to be zero for clarity.

For use in the subsequent derivation, we now take the shape derivative of Eq. (55). Terms containing the pseudo-time derivative of the adjoint fields recover the state equation of the coupled system and sum to zero. 
This leaves us with the following expression

$$
\begin{aligned}
& \int_{\Omega_{s}} \boldsymbol{\lambda}_{\epsilon}^{T} \mathcal{C} \boldsymbol{\epsilon}^{\prime} \mathrm{d} \Omega-\omega^{2} \rho_{s} \int_{\Omega_{s}} \boldsymbol{\lambda}_{u}^{T} \mathbf{u}^{\prime} \mathrm{d} \Omega \\
& +\int_{\Omega_{a}}\left(\nabla \lambda_{p}\right)^{T} \nabla p^{\prime} \mathrm{d} \Omega-\frac{\omega^{2}}{c_{a}^{2}} \int_{\Omega_{a}} \lambda_{p} p^{\prime} \mathrm{d} \Omega \\
& -\int_{\Gamma_{a s}} \boldsymbol{\lambda}_{u}^{T} \mathbf{n}_{a} p^{\prime} \mathrm{d} \Gamma-\omega^{2} \rho_{a} \int_{\Gamma_{a s}} \lambda_{p} \mathbf{n}_{a}^{T} \mathbf{u}^{\prime} \mathrm{d} \Gamma+ \\
& \int_{\partial \Omega_{s}} \mathcal{G}_{s} V_{n} \mathrm{~d} \Gamma+\int_{\partial \Omega_{a}} \mathcal{G}_{a} V_{n} \mathrm{~d} \Gamma+\int_{\Gamma_{a s}} \mathcal{G}_{a s} V_{n} \mathrm{~d} \Gamma=0
\end{aligned}
$$

where the $\mathcal{G}$ functions collect the boundary terms in Eq. (56) that are the coefficients of the normal velocity $V_{n}$ :

$$
\begin{aligned}
\mathcal{G}_{s} & =\boldsymbol{\lambda}_{\epsilon}^{T} \mathcal{C} \boldsymbol{\epsilon}-\omega^{2} \rho_{s} \boldsymbol{\lambda}_{u}^{T} \mathbf{u} \\
\mathcal{G}_{a} & =\left(\nabla \lambda_{p}\right)^{T} \nabla p-\frac{\omega^{2}}{c_{a}^{2}} \lambda_{p} p \\
\mathcal{G}_{a s} & =-\nabla\left(\boldsymbol{\lambda}_{u}^{T} \mathbf{n}_{a} p\right)^{T} \mathbf{n}_{a}-\kappa\left(\boldsymbol{\lambda}_{u}^{T} \mathbf{n}_{a} p\right) \\
& -\omega^{2} \rho_{a}\left(\nabla\left(\lambda_{p} \mathbf{n}_{a}^{T} \mathbf{u}\right)^{T} \mathbf{n}_{a}-\kappa\left(\lambda_{p} \mathbf{n}_{a}^{T} \mathbf{u}\right)\right)
\end{aligned}
$$

and $\kappa$ is the mean curvature defined in Eq. (6).

In order to construct the adjoint equation, the differentiated weak form in Eq. (56) is added to Eq. (54) and the terms containing $p^{\prime}$ and $\mathbf{u}^{\prime}$ are collected together. The adjoint variables are now chosen so that the following adjoint equation is fulfilled

$$
\begin{aligned}
& \int_{\Omega_{s}} \boldsymbol{\lambda}_{\epsilon}^{T} \mathcal{C} \boldsymbol{\epsilon}^{\prime} \mathrm{d} \Omega-\omega^{2} \rho_{s} \int_{\Omega_{s}} \boldsymbol{\lambda}_{u}^{T} \mathbf{u}^{\prime} \mathrm{d} \Omega \\
& +\int_{\Omega_{a}}\left(\nabla \lambda_{p}\right)^{T} \nabla p^{\prime} \mathrm{d} \Omega-\frac{\omega^{2}}{c_{a}^{2}} \int_{\Omega_{a}} \lambda_{p} p^{\prime} \mathrm{d} \Omega \\
& -\int_{\Gamma_{a s}} \boldsymbol{\lambda}_{u}^{T} \mathbf{n}_{a} p^{\prime} \mathrm{d} \Gamma-\omega^{2} \rho_{a} \int_{\Gamma_{a s}} \lambda_{p} \mathbf{n}_{a}^{T} \mathbf{u}^{\prime} \mathrm{d} \Gamma= \\
& -\int_{\Omega_{o b j}} \frac{\partial \Phi}{\partial p} p^{\prime} \mathrm{d} \Omega-\int_{\Omega_{o b j}} \frac{\partial \Phi}{\partial \mathbf{u}} \mathbf{u}^{\prime} \mathrm{d} \Omega
\end{aligned}
$$

Using the discretization approach outlined in Eq. 42 the discrete form of the Eq. (58) takes the form

$$
\left[\begin{array}{cc}
\left(\mathbf{K}_{s}-\omega^{2} \mathbf{M}_{s}\right) & -\mathbf{C} \\
-\rho_{a} \omega^{2} \mathbf{C}^{T} & \left(\mathbf{K}_{a}-\omega^{2} \mathbf{M}_{a}\right)
\end{array}\right]^{T}\left[\begin{array}{l}
\boldsymbol{\lambda}_{u} \\
\boldsymbol{\lambda}_{\mathbf{p}}
\end{array}\right]=\left[\begin{array}{c}
-\frac{\partial \Phi}{\partial \mathbf{u}} \\
-\frac{\partial \Phi}{\partial p}
\end{array}\right]
$$

Considering optimization problems where the parts of the acoustic boundary $\partial \Omega_{a}$ and structural boundary $\partial \Omega_{s}$ subjected to design changes are equal to the boundary of the coupled surface $\Gamma_{a s}$ and having a set of Lagrange multipliers that satisfies the adjoint equation Eq. (58), the expression for the shape derivative of the objective function $\mathcal{J}$ becomes

$\dot{\mathcal{J}}=\int_{\Gamma_{a s}} \mathcal{G}_{\Gamma_{a s}} V_{n} \mathrm{~d} \Gamma$ where

$$
\begin{aligned}
& \mathcal{G}_{\Gamma_{a s}}=\boldsymbol{\lambda}_{\epsilon}^{T} \mathcal{C} \boldsymbol{\epsilon}-\omega^{2} \rho_{s} \boldsymbol{\lambda}_{u}^{T} \mathbf{u}+\left(\nabla \lambda_{p}\right)^{T} \nabla p-\frac{\omega^{2}}{c_{a}^{2}} \lambda_{p} p \\
& -\left[\nabla\left(\boldsymbol{\lambda}_{u}^{T} \mathbf{n}_{a} p\right)^{T} \mathbf{n}_{a}+\kappa\left(\boldsymbol{\lambda}_{u}^{T} \mathbf{n}_{a} p\right)\right] \\
& -\omega^{2} \rho_{a}\left[\nabla\left(\lambda_{p} \mathbf{n}_{a}^{T} \mathbf{u}\right)^{T} \mathbf{n}_{a}+\kappa\left(\lambda_{p} \mathbf{n}_{a}^{T} \mathbf{u}\right)\right]
\end{aligned}
$$

We note that, if the radiation boundary condition is included in the acoustic domain, ie. Eq. (18), the derivation remains the same. However, the state and adjoint variables become complex variables, in which case the shape derivative of the objective function should be replaced by

$\dot{\mathcal{J}}=\int_{\Gamma_{a s}} \Re\left(\mathcal{G}_{\Gamma_{a s}}\right) V_{n} \mathrm{~d} \Gamma$

In order to convert the optimization problem with an inequality volume constraint to an unconstrained optimization problem (Nocedal and Wright 2006), the following augmented Lagrange function is proposed:

$\mathcal{L}=\mathcal{J}+\lambda\left[\int_{\Omega_{s}} \mathrm{~d} \Omega-V_{0}\right]+\frac{1}{2 \Lambda}\left[\int_{\Omega_{s}} \mathrm{~d} \Omega-V_{0}\right]^{2}$

where $\lambda$ is the Lagrangian multiplier and $\Lambda$ is a penalization parameter. The updating scheme is

$\lambda^{l+1}=\lambda^{l}+\frac{1}{\Lambda^{l}}\left[\int_{\Omega_{s}} \mathrm{~d} \Omega-V_{0}\right], \quad \Lambda^{l+1}=\eta \Lambda^{l}$

where $l$ is the number of the current iteration of the algorithm and $\eta$ is a smalll positive number $(>1)$ which slowly increases the value of the penalization parameter $\Lambda$. Thus, the shape derivative of the augmented Lagrange function reads

$\dot{\mathcal{L}}=\int_{\Gamma_{a s}} \underbrace{\left(\Re\left(\mathcal{G}_{\Gamma_{a s}}\right)+\lambda^{l}+\frac{1}{\Lambda^{l}}\left[\int_{\Omega_{s}} \mathrm{~d} \Omega-V_{0}\right]\right)}_{\mathcal{G}_{\text {tot }}} V_{n} \mathrm{~d} \Gamma$

Using the steepest decent method in which the decent direction satisfies $\dot{\mathcal{L}}<0$, the design velocity $V_{n}$ is chosen as

$V_{n}=-\mathcal{G}_{\text {tot }}$

which will be used in the update scheme presented later.

\subsection{Design update methods}

Before proceeding we note that all reviewed methods are based on an optimization cycle that consists of FE analysis, sensitivity analysis and design updates repeated in an iterative fashion until a convergence criteria is met. The procedures applied for the design updates for the density methods and the level set method will be outlined in the following. 


\subsubsection{Non-linear mathematical programming}

The topology optimization problem can be posed by first defining a real valued cost function $\Phi$. The minimization of this function with respect to the design variables $\gamma$ is sought while satisfying the given constraints $\psi_{i}$. Mathematically, the problem is stated as

$$
\begin{array}{ll}
\min _{\boldsymbol{\gamma}} & \Phi(\boldsymbol{\gamma}, \mathbf{v}(\boldsymbol{\gamma})) \\
\text { s.t. } & \mathbf{r}(\boldsymbol{\gamma}, \mathbf{v}(\boldsymbol{\gamma}))=\mathbf{S v}-\mathbf{h}=0 \\
& \psi_{i} \leq 0 \\
& 0 \leq \boldsymbol{\gamma} \leq 1
\end{array}
$$

The solution to the above stated optimization problem (Eqs. 67) to (70) ) is solved using nonlinear programming tools. A popular choice among the topology optimization community is the method of moving asymptotes (MMA) algorithm (Svanberg 1987, 2001). The algorithm requires the derivatives of both the cost function $\Phi$ and the constraints $\psi_{i}$ with respect to the design variable $\gamma$. The problem is solved in a nested manner, such that the state-problem is left out of the optimization problem. It should be noted that topology optimization problems are often characterized by a large number of design variables and few constraints.

To introduce mesh independency and to avoid checkerboard problems, regulations techniques should be applied on the design parametrization. For this purpose, either the convolution type density filtering (Bruns and Tortorelli 2001) or the density filtering based on the solution of a Helmholtz type equation (Lazarov and Sigmund 2011) is applied on the design variable field. Also to achieve crisp designs, projection schemes on the filtered design variable can be applied (Guest et al 2004; Wang et al 2011). Additionally, a continuation scheme is applied on the penalization parameter $n$ in the interpolation scheme in Eq. (36)- 36) which reduces the risk for the optimization algorithm to get stuck in a local minimum.

The overall algorithm for density based topology optimization of acoustic-structure interaction problems can be stated as follows

1. Initialize the design variable field. This can be either uniform, random or a specified design.

2. Apply filtering operation to obtain the physical design.

3. Solve the mixed state equation, Eq. (43).

4. Solve the adjoint equation, Eq. (50).

5. Calculate the sensitivity, Eq. (52), and apply chain rule to take filtering operation into account.

6. Update the design using MMA.
7. Stop iterations when the change in design variables is below a user defined tolerance or continue from the step 2 .

\subsubsection{BESO}

As an alternative to using mathematical programming for the design updates in density based topology optimization, the BESO formulation is also included in this work. The overall optimization algorithm is identical to the one presented in the previous section except for step 6 which is replaced by:

1. [6.] Heuristic design updates based on the sensitivities evaluated at the discrete design (without any intermediate densities and following a "soft-kill" approach where only structural and acoustic elements are allowed).

This means that at all stages throughout the optimization procedure the design will be fully discrete, meaning that the removed structural elements are replaced with acoustic elements and vice versa. The corresponding material properties of both media are still calculated through the interpolation functions given in the Eqs. (35) to 38. Various heuristic update schemes have been introduced for the BESO approach, see eg. Huang and Xie (2007, 2009); Huang et al (2010); Huang and Xie (2010). Here we use the BESO update scheme presented in Huang and Xie (2009) and Huang et al (2010).

The BESO formulation has previously been presented in Vicente et al (2015) for acoustic-mechanical interaction problems. However, it should be noted that our BESO implementation is based on the mixed finite element formulation (section 2.2.2), hence the sensitivities are calculated in the same fashion as presented in section 2.3.1. This means that our BESO approach differs from the one in (Vicente et al 2015) where a segregated finite element model is used to solve the acousticmechanical interaction problem and only the structural part of the domain is included in the sensitivity analysis.

\subsubsection{Boundary shape evolution}

When using the level set approach the normal "design" velocity $V_{n}$, computed such that $\dot{\mathcal{J}}<0$, is used to update the design. A Hamilton-Jacobi type of equation Osher and Fedkiw 2003) is obtained by the defining a "time derivative" of the level set function $\phi(x)$ :

$\frac{\partial \phi}{\partial t}-V_{n}|\nabla \phi|=0 \quad$ in $\Omega, \quad \phi(x, 0)=\phi_{0}(x)$ 
As seen from the Hamilton-Jacobi equation, the design $\partial \Omega$ is updated by moving the zero level set $(\phi=0)$ with the normal velocity $V_{n}$ of the moving boundaries.

Solution of the Hamilton-Jacobi, ie. Eq. (71), is most commonly realized by employing the finite difference method. A number of different explicit upwinded finite difference schemes can be found in the literature (Sethian 1999 Osher and Fedkiw 2003), which provide a robust and stable solution to Eq. (71). In a finite element framework, Xing et al (2010) realized the solution of the Hamilton-Jacobi equation by adding stabilizing diffusion in the streamline direction, whereas Liu et al (2005) solved a reaction-diffusion equation obtained by adding an artificial diffusion term.

In our implementation we make use of the finite volume method and hence solve the following form of the Hamilton-Jacobi equation

$\frac{\partial \phi}{\partial t}-\mathbf{v}_{n} \cdot \nabla \phi=0$

where $\mathbf{v}_{n}=V_{n}\left(\frac{\nabla \phi}{|\nabla \phi|}\right)$ and the discretization is done on the equivalent divergent form of Eq. 72 , which reads

$\frac{\partial \phi}{\partial t}-\left[\nabla \cdot\left(\mathbf{v}_{n} \phi\right)-\phi \nabla \cdot \mathbf{v}_{n}\right]=0$

First order upwind scheme is utilized for the discretization of the convective term and the temporal term is discretized by the finite difference method using the first order forward Euler scheme. Stable time evaluation is realized by satisfying the CFL stability condition

$\Delta t \leq \frac{h}{\max \left|V_{n}\right|}$

where $h$ is the grid size in the level set mesh.

Furthermore, after a few design iterations, too steep or flat regions can occur in the level set function which can lead to inaccurate representation of the boundaries. In order to regularize the optimization, the level set function is thus periodically re-initialized into a signed distance function by solving the re-initialization equation (Osher and Fedkiw 2003). We remark that many alternative regularization methods exist. For example, Yamada et al (2010) proposed a level set method from the concept of the phase field model which provides a perimeter constraint method to regularize the optimization problem and does not require solving the reinitialization equation throughout the optimization. The following equation is used for reinitialization of the level set function $\phi$

$\frac{\partial \phi}{\partial t}+\mathbf{s} \cdot \nabla \phi=S(\phi)$ where $\mathbf{s}=S(\phi)\left(\frac{\nabla \phi}{|\nabla \phi|}\right)$ and the sign function $S(\phi)$ is approximated as (Peng et al 1999)

$S(\phi)=\frac{\phi}{\sqrt{\phi^{2}+|\nabla \phi|^{2} h^{2}}}$

Here it is noted that $S(\phi)$ is updated at every time-step. For the finite volume discretization of the reinitialization equation, the second term on the left hand side of Eq. (76) is also written in its equivalent divergent form as

$$
\frac{\partial \phi}{\partial t}+[\nabla \cdot(\mathbf{s} \phi)-\phi \nabla \cdot \mathbf{s}]=S(\phi)
$$

For the examples considered in this work the reinitialization equation is discretized with a first order upwind scheme for the convective term and a first order forward Euler scheme for the temporal term. Selection of the utilized time step $\Delta t$ is again based on the CFL stability condition given in the Eq. (74) where $V_{n}$ is replaced by $|\mathbf{s}|$.

The overall algorithm for the level set based topology optimization of acoustic-structure interaction problems is then

1. Initialize the level set function to represent the initial design and update the mesh in the structural and acoustic domains either by marching-squares algorithm or by total re-meshing the both domains.

2. Solve the state equation (Eq. 21).

3. Solve the adjoint equation (Eq. 58).

4. Update the Lagrange multiplier according to Eq. 64.

5. Calculate the design velocity $V_{n}$ (Eq. 66) and extrapolate it to the level set mesh.

6. Solve the Hamilton-Jacobi equation (Eq.73) to evolve the shape. (See section 2.4.3)

7. Re-initialize the level set function (Eq. 77). (See section 2.4.3

8. Update the mesh from the new level set function.

9. Stop iterations when the change in the objective function is below a user defined tolerance or continue from the step 2 .

\section{Comparison of methods}

In the following sections we use our implementations of the density based and level set topology optimization methods to solve two representative topology optimization problems in vibro-acoustics. Both problems concern the minimization of the sound pressure in a prescribed objective domain, i.e.

$\mathcal{J}(p)=\int_{\Omega_{o b j}}|p| \mathrm{d} \Omega$

subject to a volume constraint. 


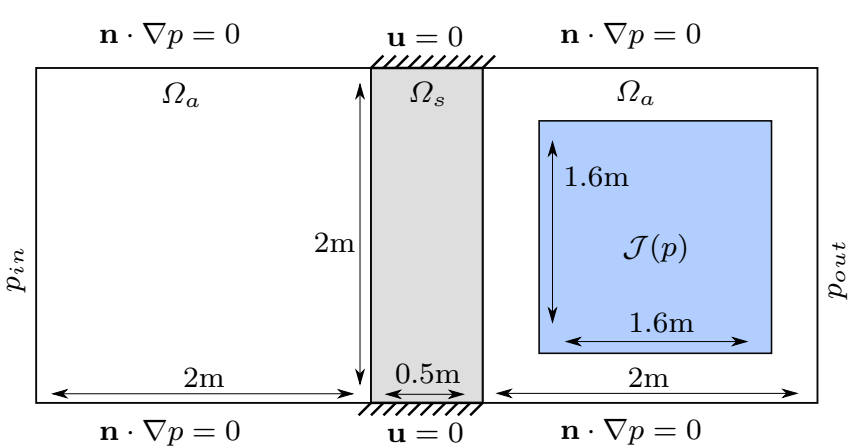

Fig. 8: Schematic illustration of the example 1 showing the boundary conditions of the optimization problem. Gray color shows the design domain, blue color illustrates the region where the objective function is evaluated.

\subsection{Example 1}

The first example is adopted from Yoon et al (2007) and a schematic illustration of the design problem, including boundary conditions, is given in the Fig. 8. The design problem concerns the design of a flexible partition which minimizes the downstream sound pressure in a duct. The model is excited by an incoming plane wave with amplitude $p_{\text {in }}=1 \mathrm{kPa}$ to the left and the right most boundary is modelled as open using an absorbing boundary condition. The optimization is carried out for a single frequency of $f=1.0 / \pi \mathrm{Hz}$. The allowed volume fraction is set to $55 \%$ of the design domain. The material properties of the considered structure and the acoustic fields are listed in the tables 11 and 2. To allow for a fair comparison, the same background mesh is used for both density based and level set topology optimization methods, i.e. an uniform mesh with an element size of $2 \times 10^{-2} \mathrm{~m}$.

\begin{tabular}{ccc}
\hline$E[\mathrm{~Pa}]$ & $\nu$ & $\rho_{s}\left[\mathrm{Kg} / \mathrm{m}^{3}\right]$ \\
\hline 1000.0 & 0.3 & 1.0 \\
\hline
\end{tabular}

Table 1: Material properties considered for the structure.

\begin{tabular}{cc}
\hline$c_{a}[\mathrm{~m} / \mathrm{s}]$ & $\rho_{a}\left[\mathrm{Kg} / \mathrm{m}^{3}\right]$ \\
\hline 1.0 & 1.0 \\
\hline
\end{tabular}

Table 2: Material properties considered for the acoustic domain.

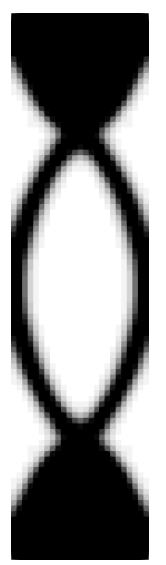

Fig. 9: Optimized design with the density based topology optimization. The objective value of the end design evaluated with mixed formulation, $\mathcal{C}=48.9 \mathrm{~N}$.

The problem is first solved using the density based formulation.To regularize the problem a Helmholtz type density filtering (Lazarov and Sigmund 2011) with a filtering radius of $r=0.015 \mathrm{~m}$ is used. Regularized Heaviside projection (Wang et al 2011) with a threshold $\eta=0$ is employed where the sharpness parameter is taken as $\beta=3$ at the start of the optimization. To avoid getting stuck in a low quality local minima we apply a continuation strategy on the convexity parameter $n$ in the RAMP interpolation functions (Eq. (36) to (38) and the sharpness parameter $\beta$. The process is started with $n=3$ and at every 50th design cycle the value is increased by one until it reaches $n=6$. After this $\beta$ takes the values of 7 and then 14 in the subsequent 50 design iterations. The initial material distribution is a uniform design with $\gamma=0.5$.

The optimized design obtained using the density method with the mixed formulation is shown in Fig. 9. The result is the well-known structure from the literature and it closely resembles the structure reported by Yoon et al (2007). We note that the problem closely corresponds to the maximization of the clamped beam's first natural frequency.

The example problem is then solved using the level set formulation. In order to avoid too large or too small gradients of the level set function we re-initialize it as a signed distance function at every 6th iteration. Also, since the level set method is known to be highly dependent on the initial topology (Villanueva and Maute 2014), we use two different initial configurations. First the design domain is initialized with a straight beam with holes distributed periodically, Fig. 10(a). Secondly, the design obtained from the density based optimization is considered as an "smart" initial guess for the level set optimization. This is included to investigate 


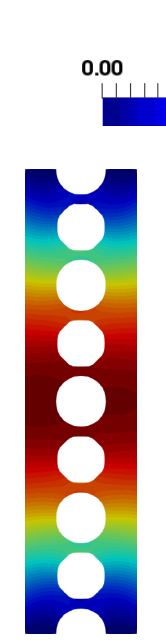

(a)

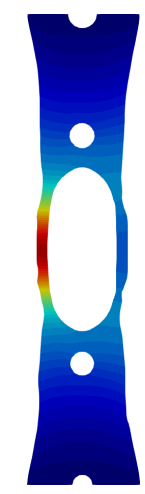

(b)

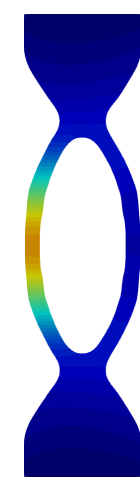

(c)

Fig. 10: Displacement magnitude $|\mathbf{u}|[\mathrm{m}]$ contours showing: (a) initial structure for the level set method, (b) optimized design obtained with the level set method, the objective value of the end $\operatorname{design} \mathcal{C}=68.7 \mathrm{~N},(\mathrm{c})$ optimized design of the level set method where the initial guess is the optimized design from the density based optimization, objective value of the end $\operatorname{design} \mathcal{C}=34.9 \mathrm{~N}$.

if the level set optimization keeps and/or improves the structure obtained using the density method.

The optimized designs are given in Fig. 10 where the displacement magnitude of the structures is included for qualitative comparison. The corresponding level set surface for the optimized design in Fig. 10(b) is shown in Fig. 14. We note that the design obtained using the density based result as initial guess performs significantly better than the design obtained with an initial configuration based on a beam with holes. Qualitatively, the design obtained using the density based method is unaltered when used as input for the level set method. However interestingly, it is found that the objective of the level set design in Fig. 10.(c) constitutes a 28.6\% reduction in objective value compared to the density based result evaluated using the mixed formulation, c.f. Fig. 9. To investigate this discrepancy further we perform a body fitted mesh analyses of all three designs using the segregated formulation. The density based design is thresholded at $\gamma=0.5$ using the marching square algorithm and the resulting frequency responses are collected in Fig. 12, From the plot it is clear that the performance of the density based design is practically identical to the best result obtained using the level set method. Hence, the discrepancy in objective value is due to the mixed formulation requiring a finer mesh than the segregated analysis (Zienkiewicz and Taylor 2000).

Fig. 11 shows the sound pressure level (SPL) of the acoustic domain. The plots confirm by visual inspection that the designs obtained by both initial guesses indeed lower the sound pressure level in the objective domain when compared to initial beam structure with holes. It is also clear that the lowest pressure level is obtained for the optimized design using the density based result as an the initial guess.

It is important to mention that the objective evolution of any initial guess for the level set method is relatively smooth, c.f. Fig. 13 . This means that the design update scheme for the level set function is likely to result in a local mimima, which is similar to solving the density based problem without a continuation scheme, i.e. using a constant high value for the penalization parameter. However, there is no obvious way to introduce the same convexification in the level set method using the Hamilton-Jacobi equation, and hence, great care must be exerted when designing the initial guess for the level set method.

\subsection{Example 2}

In Example 2 the design of a dome structure is considered. The problem is adopted from Shu et al (2014) and Vicente et al (2015) and a schematic illustration of the model problem can be seen in the Fig. 15 along with the dimensions of the computational domain. The boundary condition for the bottom of the computational domain is clamped for the structure and zero Dirichlet for the acoustic domain. All other boundary conditions for the acoustic problem are hard wall conditions. The system is excited with a point pressure load in the acoustic domain inside the dome and the objective is to minimize the absolute pressure over the prescribed objective domain near the top boundary. The design domain is fixed to the dome area shown at the Fig. 15 and $80 \%$ of the design domain is allowed to be filled with material. This example is solved for three frequencies, i.e. $4 \mathrm{~Hz}$, $5.3 \mathrm{~Hz}$ and $6 \mathrm{~Hz}$, and the sum of the absolute pressures constitutes the objective function.

\begin{tabular}{ccc}
\hline$E[\mathrm{~Pa}]$ & $\nu$ & $\rho_{s}\left[\mathrm{Kg} / \mathrm{m}^{3}\right]$ \\
\hline $100 \times 10^{3}$ & 0.3 & 100 \\
\hline
\end{tabular}

Table 3: Material properties considered for the structure. 


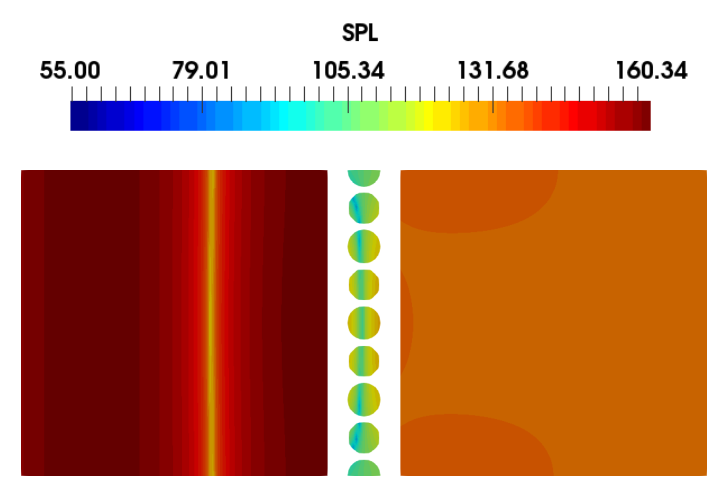

(a)

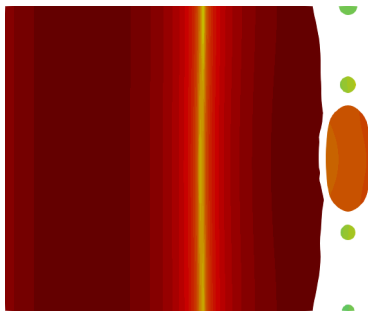

(b)

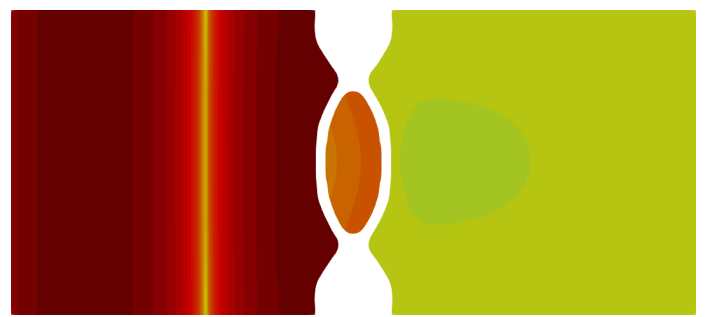

(c)

Fig. 11: Sound pressure level [dB] contours showing: (a) initial structure for the level set method, (b) optimized design obtained with the level set method, (c) optimized design of the level set method where the initial guess is the optimized design from the density based optimization.

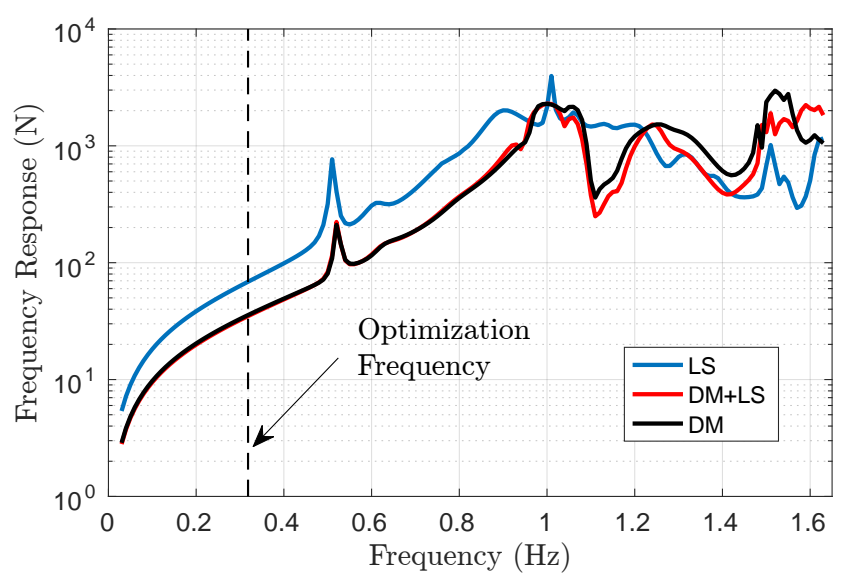

Fig. 12: Frequency response of the objective function. Black line is the optimized design with the density method (body-fitted mesh), blue line is the optimized design with the level set method and the red line is the optimized design of the level set method where the initial guess is the optimized design from the density method.

\begin{tabular}{cc}
\hline$c_{a}[\mathrm{~m} / \mathrm{s}]$ & $\rho_{a}\left[\mathrm{Kg} / \mathrm{m}^{3}\right]$ \\
\hline 343 & 1.21 \\
\hline
\end{tabular}

Table 4: Material properties considered for the acoustic domain.

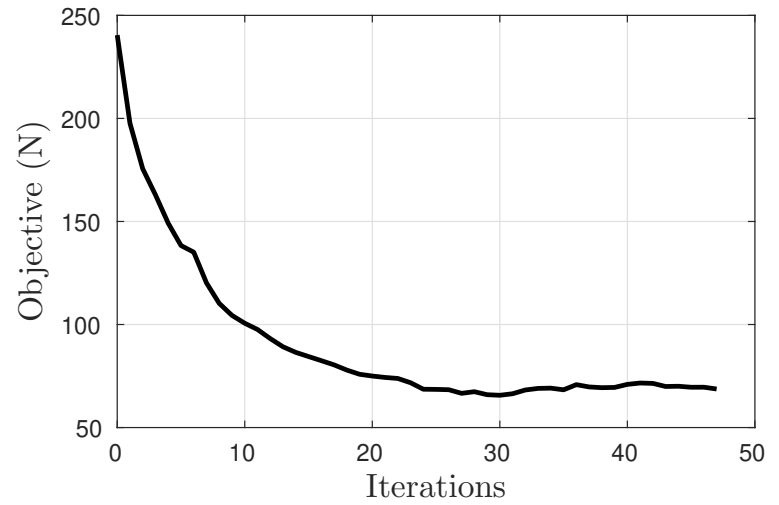

Fig. 13: The iteration history of the objective function for the level set method.

The material properties of the structure and the acoustic field are listed in the Tables 3 and 4 . We construct a uniform mesh with an element size of $1.5 \times$ $10^{-2} \mathrm{~m}$ for the computational domain and carry out the optimization using the same mesh resolution for both the density based and the level set based methods.

The density based topology optimization approach utilizes a similar continuation strategy for the convexity parameter $n$ and the sharpness parameter $\beta$ as used in Example 1, c.f. subsection 3.1. In this case, the pa- 


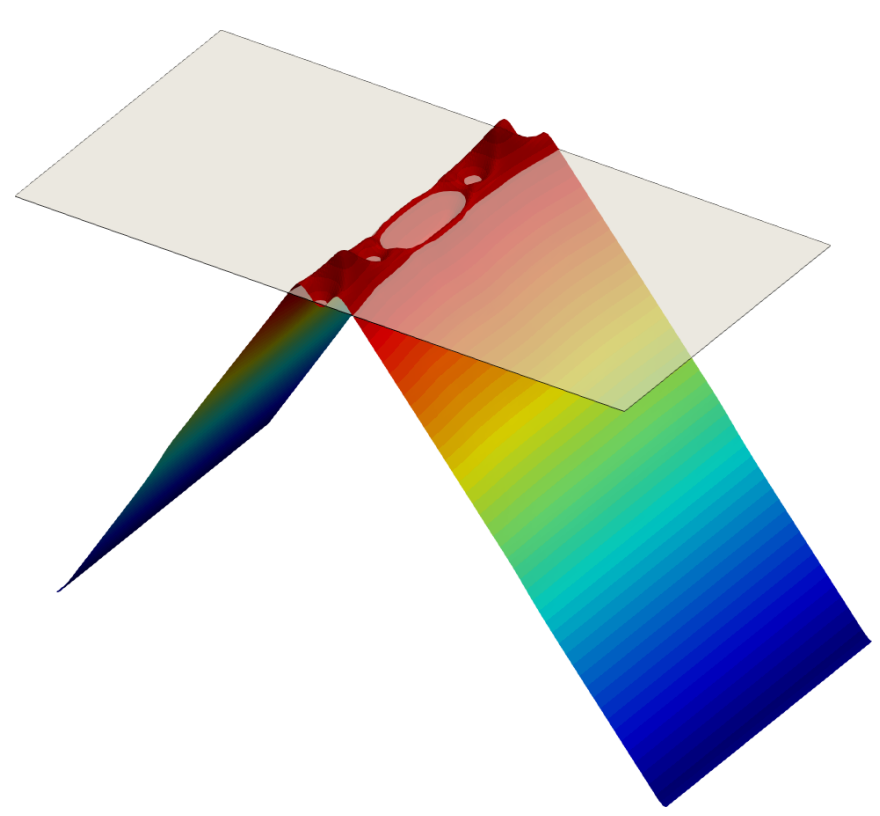

Fig. 14: Level set surface of the optimized design.

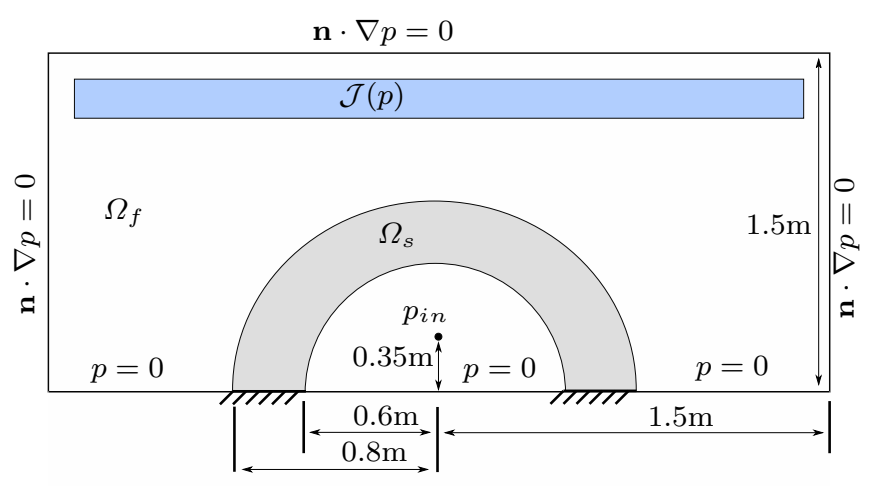

Fig. 15: Schematic illustration of example 2 showing the boundary conditions of the optimization problem. Gray color shows the design domain, blue color illustrates the region where the objective function is evaluated. The coordinates of the lower left and upper right corners of the objective domain are $(0.06,1.44)$ and $(2.94,1.485)$, respectively.

rameter $n$ is increased at every 100th design cycle and after the final value of $n=6$, the parameter $\beta$ takes the values of 7,14 and 28 for the subsequent 50 design iterations. The filtering radius and the initial material distribution are taken to be the same as in the previous example.

Fig. 16 shows the optimized design obtained using the density based method. The structure contains one partial hole at the top and the profile of the dome gets thinner towards the sides of the dome. The structure is

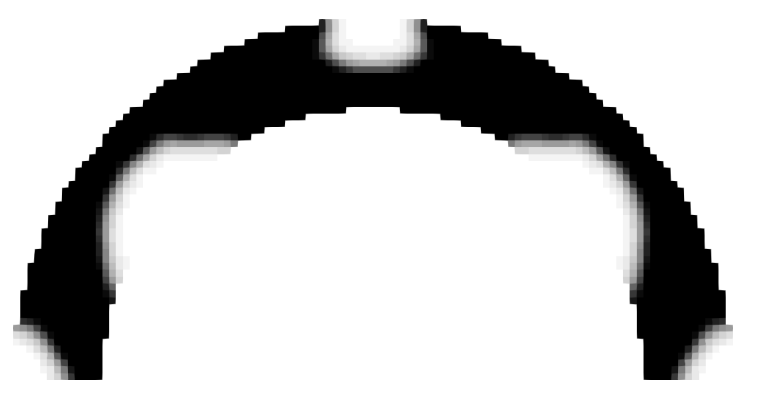

Fig. 16: Optimized design with the density based topology optimization. The objective value of the end design evaluated with mixed formulation, $\mathcal{C}=0.0022 \mathrm{~N}$.

as thick as the diameter of the prescribed design domain around the top partial hole.

The problem is then solved using the level set formulation. Similar to the first example, the level set function is re-initialized as a signed distance function every 8th iteration. In Fig. 19 the level set surface of the optimized design is shown. The optimized design contains no holes and looks very similar to the structure reported in Shu et al (2014). Although, in Shu et al (2014), the dome structure is optimized for a distributed load applied at the outer edge of the dome.

To allow for a fair comparison of the performance of the optimized designs, we once again apply a body fitted mesh analysis. In Fig. 17 we compare the displacement magnitude of the designs obtained using the density based and level set based optimization methods to the initial structure used for the level set optimization. Although the structures obtained with both optimization methods have a similar inner shape, the density based design in Fig. 17c clearly exhibits smaller displacements at the sides of the dome compared to the level set result in Fig. 17b. Similarly, the sound pressure level contours are shown in Fig. 18 clearly showing that both designs exhibit a significant reduction of the acoustic pressure outside of the dome compared to the initial dome structure with equally spaced holes. Considering the objective values of the end designs listed in Fig. 17, we note that the optimized design using the density method performs approximately $40 \%$ percent better than the structure optimized with the level set method.

Finally, the frequency responses of the dome structures are shown in Figure 20. We note that the plot consists of the three designs, i.e. the initial beam with holes and two optimization results as well as an extra design consisting of the design domain fully filled with material. Firstly, it is clear that both optimized designs outperforms the initial design and the fully filled dome. Secondly, it is noted that the optimization results in a 


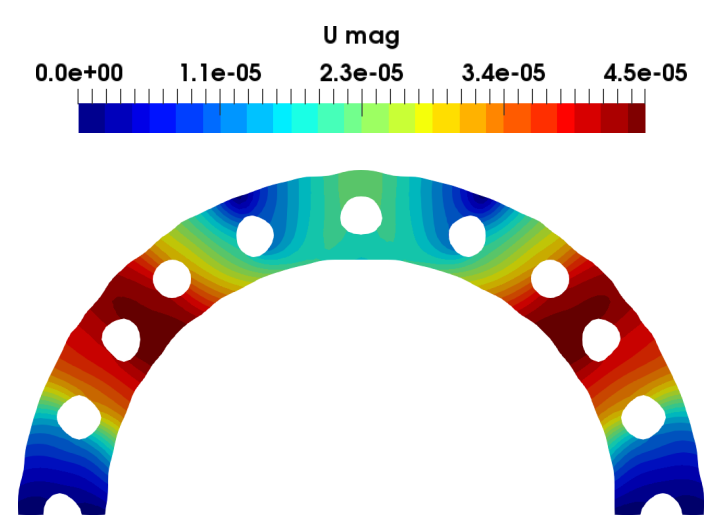

(a)

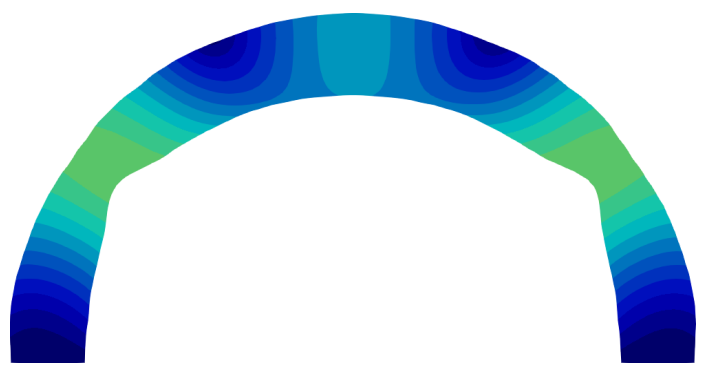

(b)

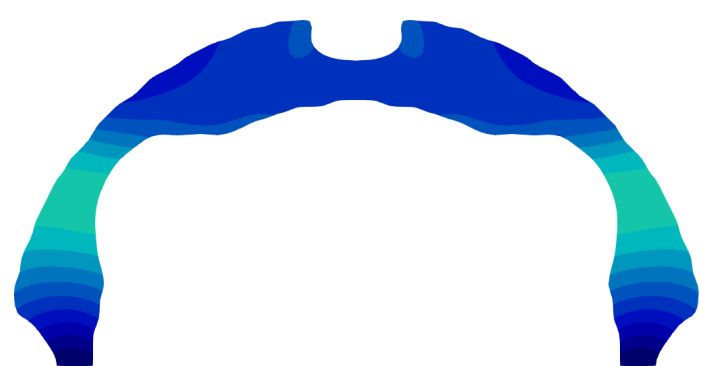

(c)

Fig. 17: Displacement magnitude $|\mathbf{u}|[\mathrm{m}]$ contours for the frequency $f=6[\mathrm{~Hz}]$ showing: (a) initial structure for the level set method, (b) optimized design obtained with the level set method, the objective value of the end design $\mathcal{C}=0.0026 \mathrm{~N}$, (c) body fitted analysis of optimized design obtained with the density method resulting in an objective value of $\mathcal{C}=0.0015 \mathrm{~N}$. The analysis in (c) is performed on a thresholded design at $\gamma=0.5$ using the marching squares algorithm.

minimization of the first resonance frequency and maximization of the second. Third and lastly, it is observed that the density based method has a better performance for two target frequencies, i.e. $f=4 \mathrm{~Hz}$ and $f=6 \mathrm{~Hz}$. However, from visual inspection of the frequency response it is clear that the level set method has the lowest response around the target frequency $f=5.3 \mathrm{~Hz}$. This advocates the use of a finer frequency range discretization though this is deemed outside the scope of the current review paper.

\subsubsection{BESO}

The comparative study is concluded with a single design obtained using the BESO formulation. The optimized design is shown in Fig. 21 along with the sound pressure level contour for a frequency of $4 \mathrm{~Hz}$. The resulting topology is found to be in good agreement with the result presented in Vicente et al (2015).

The performance of the BESO design shows a similar reduction of the acoustic pressure outside the optimized dome compared to the level set and density method results given in Fig. 18. The frequency response of the BESO optimized dome is shown in the Fig. 22 which also includes the response for the density based design, both evaluated using a body fitted mesh and a segregated analysis. The difference in response in the vicinity of the three optimization frequencies are clearly observed, showing that the density based design has superior performance for the target frequencies of $4 \mathrm{~Hz}$ and $6 \mathrm{~Hz}$, whereas the BESO design exhibits better performance around the second target frequency $5.3 \mathrm{~Hz}$ similar to the level set design. It is noted that for all methods the reduction in the sound pressure level in the specified frequency range, is obtained by reducing the first resonance frequency and increasing the second resonance frequency of the coupled system. Here it is noted that, even though the BESO approach provides a crisp design without any gray scale, the calculated objective value of the final design shows a significant discrepancy in the objective value compared to a body fitted analysis of the design, c.f. Fig. 21. This further underlines the previously mentioned inherent limitations of the standard $\mathbf{u}-p$ mixed formulation wrt. modelling accuracy. 


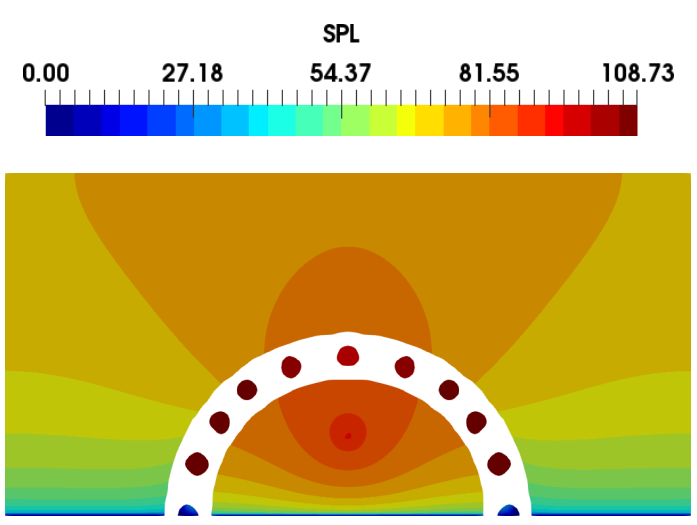

(a)

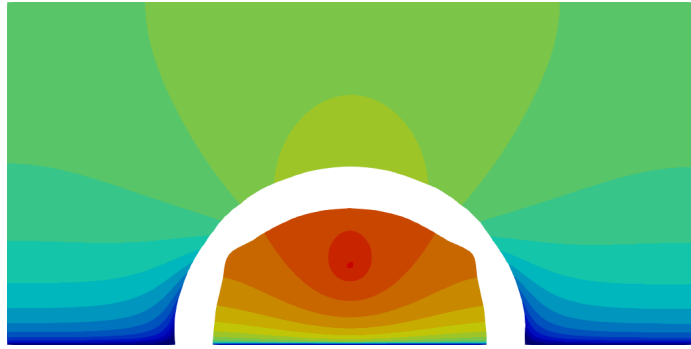

(b)

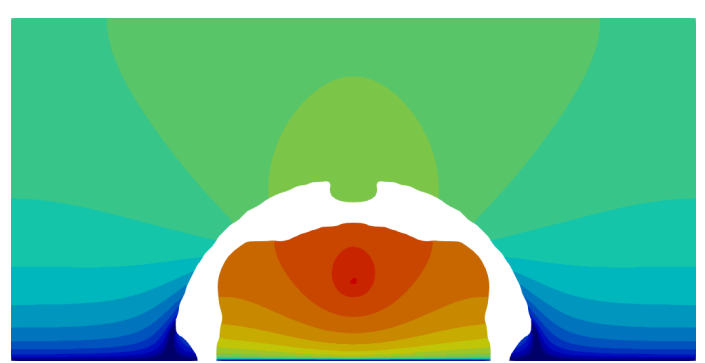

(c)

Fig. 18: Sound pressure level $[\mathrm{dB}]$ contours for the frequency $f=4[\mathrm{~Hz}]$ showing: (a) initial structure for the level set method, (b) optimized design obtained with the level set method and (c) body fitted analysis of the optimized design obtained with the density method, thresholded at $\gamma=0.5$ using the marching squares algorithm.

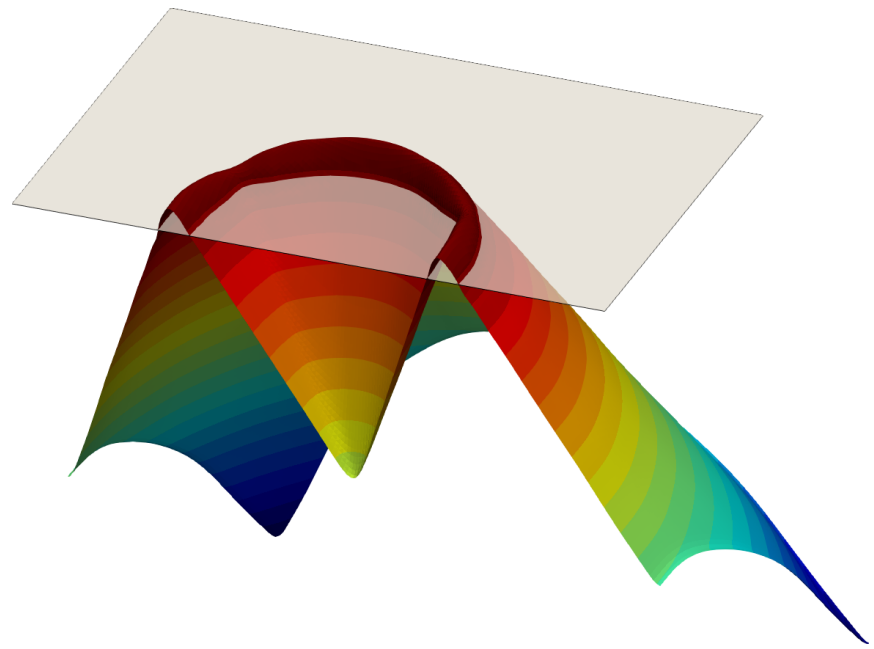

Fig. 19: Level set surface of the optimized design.

\section{Conclusions and recommendations}

The aim of this review paper has been to provide an overview and comparison of the different approaches that have currently been applied for solving topology optimization problems in vibro-acoustics. In the following we summarize the most significant findings, high-

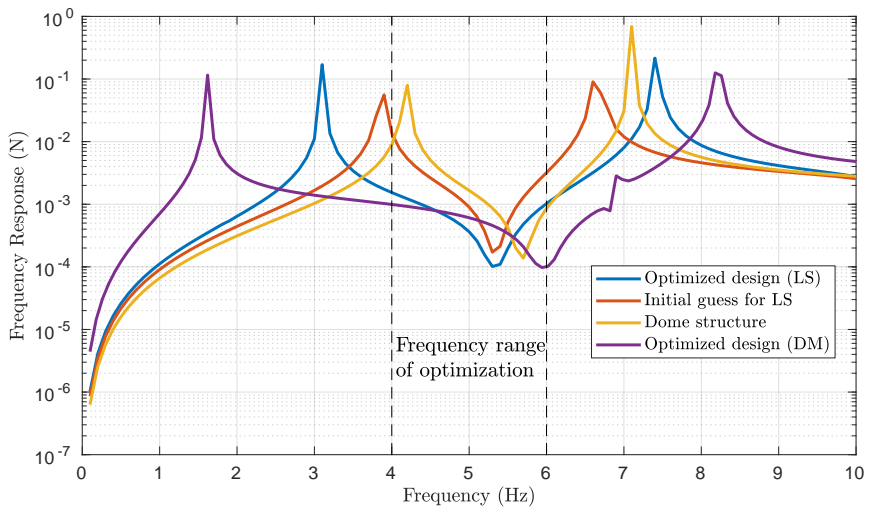

Fig. 20: Frequency response of the objective function for example 2. The blue line is the optimized design using the level set method, the red line is the initial design with holes, the yellow line is the design domain fully filled with material and the purple line is the optimized design using the density method.

light the challenges and conclude with recommendations for future directions within the field of acousticmechanical topology optimization.

For all studied examples the density based method was shown to provide the best performing designs from 


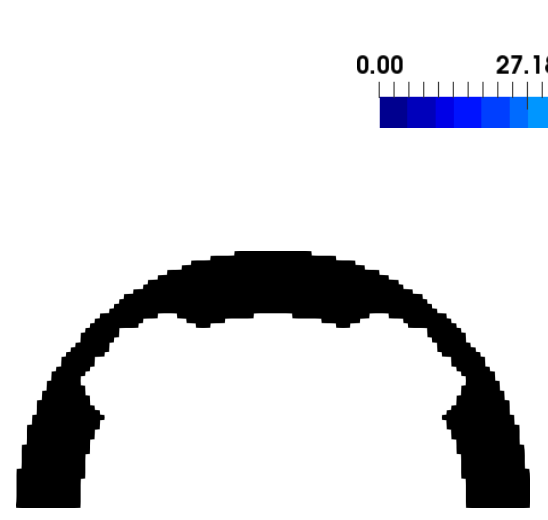

(a)

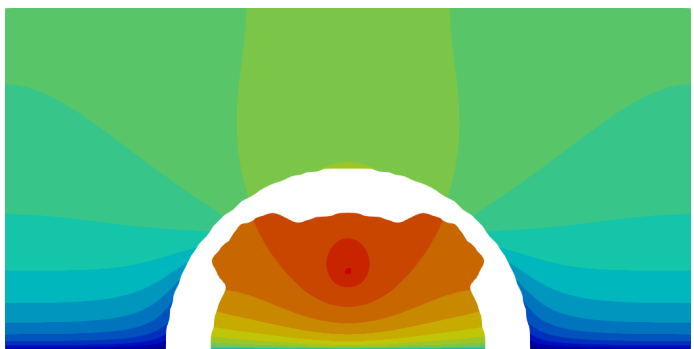

(b)

Fig. 21: Optimized design obtained from the BESO method with mixed FE formulation showing: (a) final optimized design field, (b) Sound pressure level $[\mathrm{dB}]$ contour for the frequency $f=4 \mathrm{~Hz}$. The objective value of the end design evaluated with mixed formulation, $\mathcal{C}=0.0029 \mathrm{~N}$ and body fitted analysis of optimized design obtained with BESO resulting in an objective value of $\mathcal{C}=0.0019 \mathrm{~N}$.

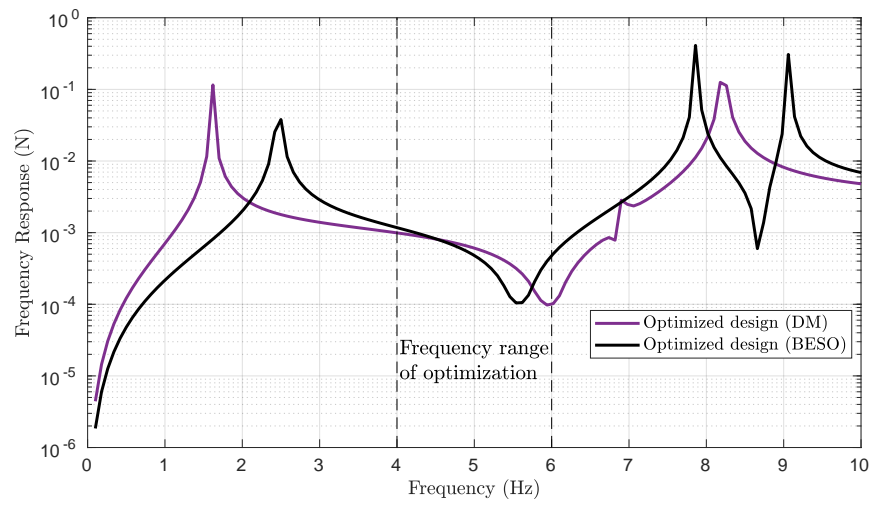

Fig. 22: Frequency response of the objective function. Black line is the optimized design obtained from BESO approach with mixed formulation, purple line is the optimized design with the density method.

an arbitrary initial guess. We mainly ascribe this to the possibility of designing a continuation scheme on the penalization parameter that effectively and consistently results in better local minima. On the contrary, the level set approach does not facilitate such a continuation scheme making the results more prone to stuck in local minima and highly dependent on the initial design. Also, though not presented here, we emphasize that the use of rigorous mathematics such as non-linear programming methods easily facilitates the inclusion of additional constraints on both physics and geometry (Sigmund and Maute 2013).

However, this should not be interpreted as a rejection of the level set based methods. On the contrary, problems having a multiphysical nature is often highly dependent on the interface representation, i.e. the cou- pling conditions. The examples presented here clearly demonstrated the issues arising from intermediate density regions and thus a poor interface representation. That is, poor accuracy in the modeling using mixed formulation makes the density based optimization approach challenging and problematic for problems which are strongly coupled and sensitive to design changes at the interface. Another significant challenge for the density based methods is that the optimized designs require a postprocessing step and a subsequent body fitted analysis to verify its performance. This step can be completely avoided when using the level set methods with a crisp interface representation, which together with the possibility to easily enforce complicated coupling conditions advocates the continued use and development of level set methods.

Finally, it was shown that the BESO method provided comparable results in the second example. While the method generally yields very good results for pure static structural optimization where the design sensitivities are of equal sign (more material is always better), its application to more complicated problems involving dynamics and/or multi-physics appears to be more problematic. Throughout the work, the BESO method is found to be the least stable of the considered methods and in some cases it leads to lack of convergence. This we ascribe to the heuristic BESO update algorithm that is not well suited for handling problems with both positive and negative design sensitivities. However, the BESO method also has its justification. That is, the method is very easy to implement in commercial blackbox codes without the need to access element integration routines and does not require expensive re-meshing schemes. In fact, for many problems one can use energy 
expression to get sensitivity information which makes it even simpler to adopt into existing codes.

\subsection{Recommendations}

Firstly, we recommend that subsequent work on vibroacoustic optimization always includes a benchmark against previous work as shown in this paper. That is, solving old problems with new methods can only be justified if the new method provides an improvement compared to existing methods. In the following we provide recommendations for the density and level set method, separately.

For the density based methods we have the following recommendations. Firstly, we note that the mixed formulation is an expensive modeling tool since it can lead to poor accuracy on coarse meshes, even with crisp designs, and that the intermediate densities at the interface lack a physical explanation. The mixed formulation is also prone to numerical instabilities arising from the choice of interpolation functions (Wang and Bathe 1997), which means that for a stable solution high order elements must be used which in turn increases the computational complexity even further. Therefore we suggest that more work should go into new interpolation schemes that, potentially, could alleviate the need for the mixed formulation. On the other hand, a monolithic formulation have many desirable properties and hence another path to follow is to modify, or expand, the standard $\mathbf{u}-p$ mixed formulation such that it is better at capturing the sharp jumps in state fields that arise when performing topology optimization.

For the level set based methods we have the following recommendations. The main issue with level set methods using the Hamilton-Jacobi update scheme is the problem of adding more constraints. We therefore suggest that focus is put on methods that ensure crisp interfaces, e.g. xFEM (Gerstenberger and Wall 2008) or CutFEM methods (Hansbo and Hansbo 2004; Burman et al 2014), together with mathematical programming tools. This latter would allow the optimization analyst to include more constraints whereas the first means that tedious post processing can be avoided.

\section{Replication of results}

All results presented in this work are in fact reproductions of already published and developed methods. For replicating the presented examples, the readers can find the relevant information in the corresponding sections.

\section{Conflict of interest}

The authors state that there is no conflict of interest.

Acknowledgements The authors would like to acknowledge the members of the DTU TopOpt group for numerous discussions and good advises on different aspects of performing multiphysical structural optimization.

\section{References}

Aage N, Andreassen E, Lazarov BS, Sigmund O (2017) Gigavoxel computational morphogenesis for structural design. Nature 550(7674):84-86, URL http://dx.doi.org/10. 1038/nature23911, letter

Akl W, El-Sabbagh A, Al-Mitani K, Baz A (2009) Topology optimization of a plate coupled with acoustic cavity. International Journal of Solids and Structures 46(10):2060 2074, DOI https://doi.org/10.1016/j.ijsolstr.2008.05.034, URL http://www.sciencedirect.com/science/ article/pii/S0020768308002096 special Issue in Honor of Professor Liviu Librescu

Alexandersen J, Sigmund O, Aage N (2016) Large scale three-dimensional topology optimisation of heat sinks cooled by natural convection. International Journal of Heat and Mass Transfer 100:876-891, DOI 10.1016/j. ijheatmasstransfer.2016.05.013

Bendsøe MP (1989) Optimal shape design as a material distribution problem. Structural optimization 1(4):193202, DOI 10.1007/BF01650949, URL https://doi.org/ 10.1007/BF01650949

Bendsøe MP, Kikuchi N (1988) Generating optimal topologies in structural design using a homogenization method. Computer Methods in Applied Mechanics and Engineering 71(2):197-224, DOI 10.1016/0045-7825(88)90086-2, URL http://linkinghub.elsevier.com/retrieve/pii/ 0045782588900862

Bourdin B (2001) Filters in topology optimization. International Journal for Numerical Methods in Engineering $50: 2143-2158$

Bruns T, Tortorelli D (2001) Topology optimization of nonlinear elastic structures and compliant mechanisms. Computer Methods in Applied Mechanics and Engineering 190(26-27):3443-3459, DOI 10.1016/S0045-7825(00) 00278-4

Burman E, Claus S, Hansbo P, Larson MG, Massing A (2014) CutFEM: Discretizing geometry and partial differential equations. International Journal for Numerical Methods in Engineering (December 2014):472-501, DOI 10.1002/ nme, URL http://arxiv.org/abs/1201.4903,1201.4903

Chen N, Yu D, Xia B, Liu J, Ma Z (2017) Microstructural topology optimization of structural-acoustic coupled systems for minimizing sound pressure level. Structural and Multidisciplinary Optimization 56(6):1259-1270, DOI 10.1007/s00158-017-1718-0

Choi K, Kim N (2005) Structural Sensitivity Analysis and Optimization 1: Linear Systems. New York: Springer

Christiansen RE, Sigmund O, Fernandez-Grande E (2015) Experimental validation of a topology optimized acoustic cavity. The Journal of the Acoustical Society of America 138(6):3470-3474, DOI 10.1121/ 1.4936905, URL http://scitation.aip.org/content/ asa/journal/jasa/138/6/10.1121/1.4936905 
Desai J, Faure A, Michailidis G, Parry G, Estevez R (2018) Topology optimization in acoustics and elastoacoustics via a level-set method. Journal of Sound and Vibration 420:73-103, DOI 10.1016/j.jsv.2018.01.032, URL http://dx.doi.org/10.1016/j.jsv.2018.01. 032https://linkinghub.elsevier.com/retrieve/pii/ S0022460X18300403

Dilgen SB, Dilgen CB, Fuhrman DR, Sigmund O, Lazarov BS (2018) Density based topology optimization of turbulent flow heat transfer systems. Structural and Multidisciplinary Optimization 57(5):1905-1918, DOI 10.1007/s00158-018-1967-6, URL https://doi.org/10. 1007/s00158-018-1967-6

Du J, Olhoff N (2010) Topological design of vibrating structures with respect to optimum sound pressure characteristics in a surrounding acoustic medium. Structural and Multidisciplinary Optimization 42(1):43-54, DOI $10.1007 / \mathrm{s} 00158-009-0477-y$

Dühring MB, Jensen JS, Sigmund O (2008) Acoustic design by topology optimization. Journal of Sound and Vibration 317(3-5):557-575, DOI 10.1016/j.jsv.2008.03.042

Düster A, Parvizian J, Yang Z, Rank E (2008) The finite cell method for three-dimensional problems of solid mechanics. Computer Methods in Applied Mechanics and Engineering 197(45):3768-3782, DOI 10.1016/j.cma.2008.02. 036

Gerstenberger A, Wall WA (2008) An eXtended Finite Element Method/Lagrange multiplier based approach for fluid-structure interaction. Computer Methods in Applied Mechanics and Engineering 197(19):1699-1714, DOI 10.1016/j.cma.2007.07.002

Guest J, Prevost J, Belytschko T (2004) Achieving minimum length scale in topology optimization using nodal design variables and projection functions. International Journal for Numerical Methods in Engineering 61(2):238254, DOI 10.1002/nme.1064

Hansbo A, Hansbo P (2004) A finite element method for the simulation of strong and weak discontinuities in solid mechanics. Computer Methods in Applied Mechanics and Engineering 193(33-35):3523-3540, DOI 10.1016/ j.cma.2003.12.041, URL http://linkinghub.elsevier. com/retrieve/pii/S0045782504000507

Huang X, Xie YM (2007) Convergent and mesh-independent solutions for the bi-directional evolutionary structural optimization method. Finite Elements in Analysis and Design 43(14):1039-1049, DOI 10.1016/j.finel.2007.06.006

Huang X, Xie YM (2009) Bi-directional evolutionary topology optimization of continuum structures with one or multiple materials. Computational Optimization and Applications 42(2):393-401, DOI 10.1007/ s00466-008-0312-0

Huang X, Xie YM (2010) A further review of ESO type methods for topology optimization. Structural and Multidisciplinary Optimization 41(5):671-683, DOI 10.1007/ s00158-010-0487-9

Huang X, Zuo ZH, Xie YM (2010) Evolutionary topological optimization of vibrating continuum structures for natural frequencies. Computers and Structures 88(5-6):357364, DOI 10.1016/j.compstruc.2009.11.011, URL http: //dx.doi.org/10.1016/j.compstruc.2009.11.011

Isakari H, Kondo T, Takahashi T, Matsumoto T (2017) A level-set-based topology optimisation for acoustic-elastic coupled problems with a fast bem-fem solver. Computer Methods in Applied Mechanics and Engineering 315:501 - 521, DOI https://doi.org/10.1016/j.cma.2016. 11.006, URL http://www.sciencedirect.com/science/
article/pii/S0045782516305187

Jensen JS (2007) Topology optimization of dynamics problems with padé approximants. International Journal for Numerical Methods in Engineering 72(13):1605-1630, DOI 10.1002/nme.2065

Jensen JS, Sigmund O (2011) Topology optimization for nano-photonics. Laser and Photonics Reviews 5(2):308 321, DOI 10.1002/lpor.201000014

Kook J, Jensen JS (2017) Topology optimization of periodic microstructures for enhanced loss factor using acoustic-structure interaction. International Journal of Solids and Structures 122-123:59-68, DOI 10.1016/j.ijsolstr. 2017.06.001

Larsen UD, Sigmund O, Bouwstra S (1997) Design and fabrication of compliant micromechanisms and structures with negative poisson's ratio. I E E E Journal of Microelectromechanical Systems 6(2):99-106, DOI 10.1109/84. 585787

Lazarov BS, Sigmund O (2011) Filters in topology optimization based on helmholtz-type differential equations. International Journal for Numerical Methods in Engineering 86(6):765-781, DOI 10.1002/nme.3072

Lee JS, Kang YJ, Kim YY (2012) Unified multiphase modeling for evolving, acoustically coupled systems consisting of acoustic, elastic, poroelastic media and septa. Journal of Sound and Vibration 331(25):5518-5536, DOI 10.1016/j.jsv.2012.07.027

Lee JS, Goransson P, Kim YY (2015) Topology optimization for three-phase materials distribution in a dissipative expansion chamber by unified multiphase modeling approach. Computer Methods in Applied Mechanics and Engineering 287:191-211, DOI 10.1016/j.cma.2015.01.011

Liu Z, Korvink J, Huang R (2005) Structure topology optimization: fully coupled level set method via femlab. Structural and Multidisciplinary Optimization 29(6):407-417, DOI 10.1007/s00158-004-0503-z, URL/https://doi.org/ 10.1007/s00158-004-0503-z

Miyata K, Noguchi Y, Yamada T, Izui K, Nishiwaki S (2018) Optimum design of a multi-functional acoustic metasurface using topology optimization based on zwicker's loudness model. Computer Methods in Applied Mechanics and Engineering 331:116 - 137, DOI https://doi.org/10.1016/j.cma.2017.11.017, URL http://www.sciencedirect.com/science/article/pii/ S0045782517303560

Niu B, Olhoff N, Lund E, Cheng G (2010) Discrete material optimization of vibrating laminated composite plates for minimum sound radiation. International Journal of Solids and Structures 47(16):2097-2114, DOI 10.1016/j.ijsolstr. 2010.04.008

Nocedal J, Wright SJ (2006) Numerical Optimization, second edition. World Scientific

Noguchi Y, Yamada T, Otomori M, Izui K, Nishiwaki S (2015) An acoustic metasurface design for wave motion conversion of longitudinal waves to transverse waves using topology optimization. Applied Physics Letters 107(22):221,909, DOI 10.1063/ 1.4936997, URL https://doi.org/10.1063/1.4936997. https://doi.org/10.1063/1.4936997

Noguchi Y, Yamada T, Yamamoto T, Izui K, Nishiwaki S (2016) Topological derivative for an acoustic-elastic coupled system based on two-phase material model. Mechanical Engineering Letters 2:16-00,246-16-00,246, DOI 10.1299/mel.16-00246

Noguchi Y, Yamamoto T, Yamada T, Izui K, Nishiwaki S (2017) A level set-based topology optimiza- 
tion method for simultaneous design of elastic structure and coupled acoustic cavity using a two-phase material model. Journal of Sound and Vibration 404:15 - 30, DOI https://doi.org/10.1016/j.jsv.2017. 05.040, URL http://www.sciencedirect.com/science/ article/pii/S0022460X17304352

Novotny AA, Sokołowski J (2013) Topological Derivatives in Shape Optimization. Springer-Verlag Berlin Heidelberg

Olhoff N, Bendsøe M, Rasmussen J (1991) On cad-integrated structural topology and design optimization. Computer Methods in Applied Mechanics and Engineering 89:259279

Osher S, Fedkiw R (2003) Level Set Methods and Dynamic Implicit Surfaces. Springer Verlag

Osher S, Sethian JA (1988) Fronts propagating with curvature-dependent speed: Algorithms based on hamilton-jacobi formulations. Journal of Computational Physics 79(1):12 - 49, DOI https://doi.org/10.1016/0021-9991(88)90002-2, URL http://www.sciencedirect.com/science/article/pii/ 0021999188900022

Park J, Wang S (2008) Noise reduction for compressors by modes control using topology optimization of eigenvalue. Journal of Sound and Vibration 315(4-5):836-848, DOI $10.1016 /$ j.jsv.2008.01.064

Pedersen NL (2000) Maximization of eigenvalues using topology optimization. Structural and Multidisciplinary Optimization 20(1):2-11, DOI 10.1007/s001580050130

Peng D, Merriman B, Osher S, Zhao H, Kang M (1999) A pde-based fast local level set method. Journal of Computational Physics 155(2):410-438, DOI 10.1006/jcph.1999. 6345

Picelli R, Vicente WM, Pavanello R, Xie YM (2015) Evolutionary topology optimization for natural frequency maximization problems considering acoustic-structure interaction. Finite Elements in Analysis and Design 106:56-64, DOI 10.1016/j.finel.2015.07.010

Sethian J, Wiegmann A (2000) Structural Boundary Design via Level Set and Immersed Interface Methods. Journal of Computational Physics 163(2):489-528, DOI 10.1006/ jcph.2000.6581, URL http://linkinghub.elsevier.com/ retrieve/pii/S0021999100965811

Sethian JA (1999) Level Set Methods and Fast Marching Methods: Evolving Interfaces in Computational Geometry, Fluid Mechanics, Computer Vision, and Materials Science, 2nd edn. Cambridge Monograph on Applied and Computational Mathematics, Cambridge Univeristy Press, URL http://math.berkeley.edu/ \{\}sethian/ 2006/Publications/Book/2006/book_1999.html

Shu L, Yu Wang M, Ma Z (2014) Level set based topology optimization of vibrating structures for coupled acousticstructural dynamics. Computers and Structures 132:3442, DOI 10.1016/j.compstruc.2013.10.019, URL http:// dx.doi.org/10.1016/j.compstruc.2013.10.019

Sigmund O (2001) Design of multiphysics actuators using topology optimization - part i. Computer Methods in Applied Mechanics and Engineering 190(49-50):6577-6604, DOI 10.1016/S0045-7825(01)00251-1

Sigmund O, Clausen PM (2007) Topology optimization using a mixed formulation: An alternative way to solve pressure load problems. Computer Methods in Applied Mechanics and Engineering 196(13-16):1874-1889, DOI 10.1016/j.cma.2006.09.021

Sigmund O, Maute K (2013) Topology optimization approaches: A comparative review. Structural and Multidisciplinary Optimization 48(6):1031-1055, DOI 10.1007/ s00158-013-0978-6

Søndergaard MB, Pedersen CBW (2014) Applied topology optimization of vibro-acoustic hearing instrument models. Journal of Sound and Vibration 333(3):683-692, DOI 10.1016/j.jsv.2013.09.029, URL http://dx.doi.org/10. $1016 / j . j \mathrm{jv} .2013 .09 .029$

Stolpe M, Svanberg K (2001) An alternative interpolation scheme for minimum compliance topology optimization. Structural and Multidisciplinary Optimization 22(2):116124, DOI 10.1007/s001580100129

Svanberg K (1987) The method of moving asymptotes - a new method for structural optimization. International Journal for Numerical Methods in Engineering 24(2):359-373, DOI 10.1002/nme.1620240207

Svanberg K (2001) A class of globally convergent optimization methods based on conservative convex separable approximations. Siam Journal on Optimization 12(2):555-573, DOI 10.1137/S1052623499362822

Vicente WM, Picelli R, Pavanello R, Xie YM (2015) Topology optimization of frequency responses of fluid-structure interaction systems. Finite Elements in Analysis and Design 98:1-13, DOI 10.1016/j.finel.2015.01.009

Villanueva CH, Maute K (2014) Density and level setxfem schemes for topology optimization of 3-d structures. Computational Mechanics 54(1):133-150, DOI $10.1007 / \mathrm{s} 00466-014-1027-\mathrm{z}$

Villanueva CH, Maute K (2017) Cutfem topology optimization of 3d laminar incompressible flow problems. Computer Methods in Applied Mechanics and Engineering 320:444-473, DOI 10.1016/j.cma.2017.03.007

Wang F, Lazarov BS, Sigmund O (2011) On projection methods, convergence and robust formulations in topology optimization. Structural and Multidisciplinary Optimization 43(6):767-784, DOI 10.1007/s00158-010-0602-y, URL https://doi.org/10.1007/s00158-010-0602-y

Wang X, Bathe KJ (1997) Displacement/pressure based mixed finite element formulations for acoustic fluidstructure interaction problems. International Journal for Numerical Methods in Engineering 40(11):2001-2017, DOI 10.1002/(SICI)1097-0207(19970615)40:11<2001:: AID-NME152 $>3.0 . \mathrm{CO} ; 2-\mathrm{W}$

Xing X, Wei P, Wang MY (2010) A finite element-based level set method for structural optimization. International Journal for Numerical Methods in Engineering 82(7):805842, DOI $10.1002 / \mathrm{nme} .2785$

Yamada T, Izui K, Nishiwaki S, Takezawa A (2010) A topology optimization method based on the level set method incorporating a fictitious interface energy. Computer Methods in Applied Mechanics and Engineering 199(45):2876 - 2891, DOI https://doi.org/10.1016/j. cma.2010.05.013, URL http://www.sciencedirect.com/ science/article/pii/S0045782510001623

Yamamoto T, Maruyama S, Nishiwaki S, Yoshimura M (2008) Thickness optimization of a multilayered structure on the coupling surface between a structure and an acoustic cavity. Journal of Sound and Vibration 318(1-2):109-130, DOI 10.1016/j.jsv.2008.04.004

Yamamoto T, Maruyama S, Nishiwaki S, Yoshimura M (2009) Topology design of multi-material soundproof structures including poroelastic media to minimize sound pressure levels. Computer Methods in Applied Mechanics and Engineering 198(17-20):1439-1455, DOI 10.1016/j.cma. 2008.12.008, URL http://dx.doi.org/10.1016/j.cma. 2008.12.008

Yang R, Du J (2013) Microstructural topology optimization with respect to sound power radiation. Structural 
and Multidisciplinary Optimization 47(2):191-206, DOI 10.1007/s00158-012-0838-9

Yoon GH, Jensen JS, Sigmund O (2007) Topology optimization of acoustic-structure interaction problems using a mixed finite element formulation. International Journal for Numerical Methods in Engineering 70(9):10491075, DOI 10.1002/nme.1900, URL http://doi.wiley. com/10.1002/nme.1900

Yoon GH, Choi H, Hur S (2018) Multiphysics topology optimization for piezoelectric acoustic focuser. Computer Methods in Applied Mechanics and Engineering 332:600623, DOI 10.1016/j.cma.2017.12.002

Zhang X, Kang Z (2013) Topology optimization of damping layers for minimizing sound radiation of shell structures. Journal of Sound and Vibration 332(10):2500-2519, DOI 10.1016/j.jsv.2012.12.022, URL http://dx.doi.org/10. $1016 / \mathrm{j} \cdot \mathrm{jsv} \cdot 2012.12 .022$

Zienkiewicz O, Taylor R (2000) The finite element method. Butterworth Heinemann, 